\title{
A phenotypic screen using splitCas9 identifies essential genes required for actin regulation during host cell egress and invasion by Toxoplasma gondii
}

Wei Li ${ }^{1 \#, J a n e s s a ~ G r e c h ~}{ }^{1 \#}$,Johannes Felix Stortz ${ }^{2,3 \#, M a t t h e w ~ G o w ~}{ }^{1}$, Javier Periz ${ }^{1}$, Markus Meissner $^{1,2 *}$ and Elena Jimenez-Ruiz ${ }^{1 *}$

${ }^{1}$ Experimental Parasitology, Department of Veterinary Sciences, Faculty of Veterinary Medicine, Ludwig-Maximilians-Universität, LMU. Munich, Germany.

${ }^{2}$ Wellcome Centre for Integrative Parasitology, Institute of Infection, Immunity \& Inflammation, Glasgow Biomedical Research Centre, University of Glasgow, Glasgow, United Kingdom.

${ }^{3}$ Max Planck Institute for Biology of Ageing, Cologne, Germany

* Corresponding authors: M.M.: Markus.meissner@para.vetmed.uni-muenchen.de ; E.J.R.: Elena.jimenez@para.vetmed.uni-muenchen.de

\# Equal contribution 
1 Abstract

2 Apicomplexan parasites, such as Toxoplasma gondii, possess unique organelles, cytoskeletal

3 structures, signalling cascades, replicate by internal budding within a specialised compartment

4 and actively invade and exit the host cell, to name a few aspects of the unique biology that

5 characterise this phylum. Due to their huge phylogenetic distance from well established model organisms, such as opisthokonts, comparative genomics has a limited capacity to infer gene

7 functions and conserved proteins can fulfil different roles in apicomplexans. Indeed, 8 approximately $30 \%$ of all genes are annotated as hypothetical and many had a crucial role 9 during the asexual life cycle in genome-wide screens. While the current CRISPR/Cas9-based screens allow the identification of fitness conferring genes, only little information about the respective functions can be obtained. To overcome this limitation, and to group genes of interest into functional groups, we established a conditional Cas9-system in $T$. gondii that allows phenotypic screens. Using an indicator strain for F-actin dynamics and apicoplast segregation, we identified critical genes required for defined steps during the asexual life cycle. The detailed characterisation of two of these candidates revealed them to be critical for host cell egress and invasion and to act at different time points in the disassembly of the intravacuolar F-actin network. While the signalling linking factor (SLF) is an integral part of a signalling complex required for early induction of egress, a novel conoid protein (conoid gliding protein, CGP) acts late during egress and is required for the activation of gliding motility. 


\section{Main}

Apicomplexans are early branching eukaryotes related to ciliates and dinoflagellates, with unique adaptations to an intracellular, parasitic existence. The huge phylogenetic distance to well established model organisms is also reflected by the fact that many genes are unique and annotated as hypothetical. With the advancement of the CRISPR/Cas9 technology in T. gondii, genome-wide screens allowed the identification of genes that are important during the asexual stage of the parasite ${ }^{1}$ with many hypothetical genes being critical for the survival of the parasite. While pooled screens allow the identification of fitness conferring genes, downstream assays are required to define functional groups based on the specific phenotype caused by their deletion.

For the adaptation of phenotypic screens in $T$. gondii, we previously attempted to implement a conditional Cas9 system based on $\mathrm{ddFKBP}^{2}$ and, while it was possible to identify genes critically involved in nuclear mRNA export, the ddFKBP-system ${ }^{3}$ suffered from inefficient regulation, prohibiting tight temporal control of Cas9 activity ${ }^{2}$. This resulted in non-essential genes being rapidly lost in a transfected pool (unpublished).

Here, we adapted a tight control system for Cas9, based on splitCas9 (sCas9) ${ }^{4}$. We generated indicator parasites, expressing chromobodies directed against F-actin ${ }^{5}$ and a marker for the apicoplast (FNR-RFP; a plastid like organelle) that allowed the identification of specific phenotypes in an image-based screen for parasites with abnormal invasion, replication and host cell egress and in parallel the characterisation of changes in F-actin dynamics and apicoplast segregation. We screened a library of 320 genes, with more than $40 \%$ annotated as hypothetical and conserved within the phylum of Apicomplexa ${ }^{1}$. Parasite mutants were grouped into defined phenotypes, focusing on changes in F-actin dynamics and delays in host cell egress. We identified several genes critical for host-cell invasion, replication and egress. Among them, we identified a novel conoid associated protein (TGGT1_240380) and a putative neurotransmitter symporter (TGGT1_208420) that are critical for distinct, independent steps during host cell egress and act at different times. Since, both proteins are highly conserved 
1 within apicomplexan parasites, they likely fulfil conserved, critical functions during host cell

2 egress and invasion.

3

4

\section{Results}

\section{Adaptation of a conditional Cas9-system in T. gondii}

In order to achieve tight, temporal control of Cas9, we chose the sCas9 system ${ }^{4}$, where the Cas9 enzyme is split into two subunits ( $\mathrm{N}$ - and $\mathrm{C}$-terminus), which are fused to a FKBP and FRB domain. Upon addition of rapamycin these subunits interact and Cas 9 activity is restored (Fig.1a). We generated transgenic $\mathrm{RH} \Delta H X$ parasites expressing both sCas9 subunits (Supplementary Fig.1a,b), resulting in the recipient strain $\mathrm{RHsCas9.}$

To test efficiency and specificity of this system, we generated vectors for stable expression of small guide RNAs (sgRNA) against previously described essential and non-essential genes, such as $\operatorname{sag}^{16}$ and gap $40^{7}$. These vectors were randomly integrated into the genome of $\mathrm{RHsCas} 9$ and into $\mathrm{RH} \Delta H X$ parasites (Supplementary Table1; Supplementary Fig.1c,d,e).

Subsequently, parasites were induced with $50 \mathrm{nM}$ rapamycin for $1 \mathrm{~h}$ or $48 \mathrm{~h}$ before they were analysed. Upon rapamycin treatment, a gap40-like phenotype in RHsCas9-gap40 parasites, as described in the literature ${ }^{7}$, was observed in up to $95 \%$ of vacuoles, but not in $\mathrm{RH}$-gap40 or RHsCas9 strains (Fig.1b,c). No difference in induction rate was observed upon 1 or 48 hours of rapamycin treatment, demonstrating that the sCas9 system allows efficient and rapid activation (Fig.1c).

A second control experiment targeted the non-essential surface antigen $1(\mathrm{SAG} 1)^{6}$. While $>95 \%$ of parasites demonstrated loss of SAG1, we found that up to $50 \%$ of parasites also showed aberrant morphology of their nuclei in addition to loss of SAG1 (Fig.1d,e). This phenotype was only present in rapamycin-treated parasites co-expressing sCas 9 and sag1 sgRNA. No impact on parasite morphology was observed in RHsCas9 parasites (cultured with or without rapamycin), indicating that this phenotype is not caused by general toxicity of sCas 9 (Fig.1e). We reasoned that the introduction of a double strand break (DSB) in the genome leads to a replication defect in a subpopulation of induced parasites. 


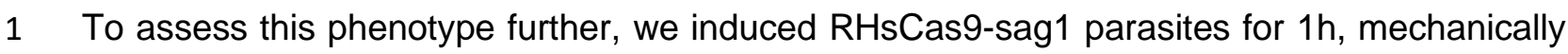

2 released them from the host cell $48 \mathrm{~h}$ later and allowed them to infect fresh HFF cells, thus

3 representing the $2^{\text {nd }}$ lytic cycle. Most of these parasites were negative for SAG1 $(79 \% \pm 4.8)$, and no aberrant morphology or nuclei were detectable (Fig.1e). To address if the nuclear phenotype is caused by the introduction of a DSB, we isolated RHsCas9-sag1KO and confirmed specific gene disruption (Supplementary Fig.2a). Next, we silently mutated sag1 into a non-cleavable copy of sag1 (sag1*) and introduced it into RHsCas9-sag1KO and noninduced RHsCas9-sag1 parasites. (Supplementary Fig.2b). Upon induction with rapamycin, we found no reduction of SAG1-positive parasites could be observed, since both strains expressed the non-cleavable sag1 $1^{*}$. However, the parasite strain with intact endogenous sag1 (RHsCas9-sag1) demonstrated a similar aberrant phenotype as described above, while no phenotype could be observed in case of RHsCas9-sag1 $1 \mathrm{KO}$ containing only the non-cleavable sag $1^{*}$ (Supplementary Fig.2c,d). This demonstrates that the introduction of a DSB can cause an aberrant nuclear phenotype.

In summary, the sCas9 system allows the efficient generation of conditional mutants, but one needs to ensure that the phenotype of interest is sufficiently distinct from the aberrant nuclear (non-specific) phenotype (Fig.1d,e) that needs to be subtracted in the readout of any screen.

Generation of an indicator parasite line for a forward genetic screen for F-actin and egress mutants.

To assess if specific phenotypes related to changes in F-actin dynamics and apicoplast replication can be identified, we tested a sCas9 strain expressing the actin Chromobody CBEmeraldFP $(\mathrm{CbEm})^{5}$ by disrupting sag1, as negative control, $d r p A^{8}$, which should only affect apicoplast division, act $1^{5,9}, a d f^{10}$ and formin 2 (frm2) ${ }^{11}$ (Fig.1f,g and Supplementary Fig.1g), which should cause different effects on F-actin dynamics and apicoplast division. For the phenotypic screen, we included the apicoplast marker, FNR-RFP ${ }^{12}$ (Fig.1h), to facilitate image acquisition. 
1 Stable transfection with the respective sgRNA expression vectors and disruption of the target

2 gene was validated by sequencing of the respective loci after gene disruption (Supplementary

3 Fig.1g). Parasitophorous vacuoles (PV) containing parasites with abnormal nuclei, representing the non-specific phenotype shown above, were excluded from subsequent analysis. As expected, disruption of sag1 or $\operatorname{drpA}$ had no detectable effect on the formation of the intravacuolar F-actin network (Fig.1g) and a strong defect in apicoplast segregation was observed in the case of $d r p A$-disruption, as previously described ${ }^{8}$. In contrast, disruption of act1 and adf led to disintegration and stabilisation of the intravacuolar network respectively (Fig.1g) as described ${ }^{5,9,13}$. In addition, disruption of the actin nucleator FRM2 also led to stabilisation of the intravacuolar network and abrogation of the intracellular polymerisation centre, replicating the effect observed when excising the locus using a DiCre strategy ${ }^{11}$. In all cases, apicoplast inheritance was affected as expected. Therefore, the indicator strain allows the identification of specific phenotypes to apicoplast maintenance, actin dynamics, or both.

Finally, we assessed if delays in parasite egress can be identified in order to screen for genes required for host cell egress (Supplementary Fig.3). In good agreement with the literature, disruption of adf and act1 led to a significant delay in egress, while the behaviour of parasites with disrupted sag1 was similar to WT parasites (Supplementary Fig.3a,b,c).

\section{Phenotypic screening using a custom-designed sgRNA library.}

For the phenotypic screen, we curated a library containing hypothetical genes without a signal peptide that are conserved in apicomplexans and have been hypothesised to be fitness conferring (phenotypic score $<-1.5)^{1}$. In total 320 genes were targeted (Supplementary Table2). As internal controls sgRNAs targeting gap40 (parasite replication ${ }^{7}$ ) and prf (F-actin dynamics and egress ${ }^{14}$ ) were included (Supplementary Fig.4a,b).

Synthesised sgRNAs were cloned into a vector containing the DHFR selection cassette as previously described ${ }^{15}$. The number of bacteria in the recovered sample carrying ligated plasmid was estimated to be $3 \times 10^{6} \mathrm{cfu}$, exceeding the number recommended to maintain 
1 complexity ${ }^{15}$. Library complexity was confirmed by sequencing sgRNA from 35 colonies picked

2 at random (not shown), out of which we identified 29 unique gRNAs (83\%).

3 Next, the library was transfected into the indicator strain, followed by drug selection, and sorting

4 of single parasites into ten 96-well plates (Fig.2a).

5 We obtained 608 clonal parasites that were inoculated onto three replica plates. Two sets were

6 induced with rapamycin for 48 and $72 \mathrm{~h}$, followed by fixation and automated imaging. Images

were independently analysed twice by different authors and graded by the relative strength of phenotypes (Fig.2b,c). From this analysis, a total of 267 clones, were put forward for analysis of integrated sgRNA. We dismissed clones with integration of multiple different sgRNAs, which caused "super-aberrant" phenotypes (Supplementary Table3, Supplementary Fig.4c). Importantly, our internal controls were isolated multiple times (Supplementary Table3, Supplementary Fig.4a,b), demonstrating the robustness of this screening approach. This procedure resulted in a total of 99 candidate genes (Supplementary Table3) that were prioritised by re-analysing the obtained images. We focused on clones with the highest induction rates and excluded candidates with strong replication defect (see above; Fig.2b,c). In summary, disruption of 42 genes showed detectable differences in F-actin formation, apicoplast segregation and/or host cell egress (Supplementary Table4). However, it should be noted that F-actin changes were relatively minor, when compared to the internal control (profilin) (Supplementary Fig.4a) or the effects seen upon disruption of adf or frm2 (Fig.1h).

We succeeded in tagging 12 of the 16 candidates classified as F-actin and/or apicoplast phenotype (Supplementary Fig.5). Interestingly, one of these genes, TGGT1_208420, localised to the intravacuolar network and the apical pole of the parasite and was also found as important for natural egress (see below; Fig.3a and Supplementary Fig.7a).

\section{Selection of candidates potentially involved in parasite egress}

Next, parasite egress was analysed $72 \mathrm{~h}$ after inoculation and induction of sCas9. At this time point, most initially infected host cells were lysed and parasites reinvaded neighbouring host cells (Fig.2c). We identified 33 clones with a potential delay in host cell egress (Supplementary 
1 Table 4). Upon a second round of visual inspection, parasites forming smaller or aberrant PVs,

2 indicating obvious replication defects, were excluded, resulting in the identification of 3 candidates where conditional disruption resulted in a delayed egress phenotype 4 (Supplementary Table4).

5 Analysis of stimulated egress using calcium ionophore A23187 (Ci A23187) revealed that disruption of 4 candidate genes resulted in significantly delayed egress. In contrast, control parasites with disrupted act1 and adf, were inhibited in egress (Supplementary Fig.3a and Supplementary Fig.6a,b). Since disruption of the gene TGGT1_252465, resulted in slower replication, it was excluded from additional analysis (Supplementary Fig.6c). Finally, the 3 remaining candidates also demonstrated a significant reduction in host cell invasion rates when compared to controls (Supplementary Fig.6d). One candidate (TGGT1_248640) has recently been described as one of the non-discharge proteins, $\operatorname{TgNd}^{16}$.

Here we focus our analysis on the remaining 2 candidates, TGGT1_240380 and TGGT1_208420, named conoid gliding protein (CGP) and signalling linker factor (SLF) respectively (Fig.2d).

\section{CGP and SLF are required for egress and invasion}

We tagged both genes C-terminally with Halo-Tag (cgp-Halo) or mCherry (slf-mCherry or slfHalo) (Fig.3a, Supplementary Fig.7a). Both proteins showed an apical localisation, as evidenced via colocalisation with the apical IMC-marker ISP ${ }^{17}$. In addition, SLF also accumulated in the intravacuolar network that connects individual parasites within the PV5 (Supplementary Fig.9a).

To validate the phenotype seen with sCas9, genes were floxed in the RHDiCre $\Delta K u 80$ strain ${ }^{18}$ (Fig.3b; Supplementary Fig.7, Fig.8a and Fig.9b) to generate conditional null mutants (cKO). Induction of DiCre using rapamycin led to a severe growth defect, as evidenced by plaque assays, confirming their critical role during the asexual life cycle of the parasite (Fig.3c). Further analysis fully confirmed the phenotype obtained using sCas9 and demonstrated a crucial function of both proteins in host cell egress and invasion, while parasite replication, 
1 morphology, apicoplast and secretory organelles were not affected (Fig.3d,e;

2 Supplementary Fig.8b,d,e,f and Fig.9c,d,g,h,i).

3 Gliding motility was significantly reduced in both mutants (Fig.3f; Supplementary Fig.8c,

4 Fig.9e; Movie_S1). Interestingly, in the case of parasites lacking slf (s/f-cKO) gliding motility and egress (Fig.3e; Supplementary Fig.9e,f) could be partially rescued upon addition of $\mathrm{Ci}$ A23187 (Supplementary Fig.9f; Movie_S2), indicating that it is involved in a signalling cascade, upstream of intracellular calcium release. Indeed, slf-cKO showed a significantly reduced secretion of micronemes (Fig.3g), that can be partially rescued upon addition of Ci A23187, but not BIPPO ${ }^{19}$. Therefore, the phenotype of slf-cKO appeared similar to the phenotypes observed upon deletion of components of the phosphatidic acid (PA) signalling platform, such as diacylglycerol kinase 2 (DGK2), cell division control 50 related protein (CDC50.1), guanylate cyclase (GC) or unique GC organiser $(\mathrm{UGO})^{20}$.

In the case of cgp knockout parasites (cgp-cKO), egress was blocked irrespective of addition of Ci A23187, BIPPO or Propanolol (Fig.3e). Furthermore, no defects of microneme secretion could be observed (Fig.3g), placing this protein into a different functional category.

Analysis of host cell egress reveals that CGP and SLF act at two distinct, temporally controlled steps.

We then characterised the sequential action of CGP and SLF during host cell egress. The intravacuolar network is rapidly disintegrated early in the egress process ${ }^{5}$, thereby acting as an early indicator for initiation of host cell egress. Therefore, we introduced $\mathrm{CbEm}^{5}$ into the UPRT locus of both mutants to analyse localisation and dynamics of F-actin (Fig.4a; Supplementary Fig.7k,I; Movies_S3, S4 and S5).

When analysing F-actin dynamics upon induction of egress, the following steps can be differentiated in WT parasites: 1) disassembly of the intravacuolar network, 2) reduction of F-actin nucleation close to the Golgi, probably caused by $\mathrm{FRM}^{5}$ ) activation of motility and strong posterior accumulation of F-actin (Fig.4a,b). In the case of slf-cKO parasites, none of these steps could be observed indicating that initiation of egress is completely blocked and 
1 that the initial signals leading to the induction of microneme secretion are identical with

2 regulation of F-actin dynamics during host cell egress (Fig.4a,c; Movie_S5). This phenotype

3 was partially rescued upon addition of Ci A23187. (Movie_S4).

Interestingly, induction with propranolol led to a different egress phenotype, when compared to induction with BIPPO. Here the parasites that remained within the PVM were able to disassemble the filaments but appeared unable to initiate motility (Fig.4a; Movie_S4).

In contrast, deletion of cgp led to a block in a later stage during egress. While disassembly of the intravacuolar network and reduction of F-actin nucleation close to the Golgi occurred normally, neither posterior accumulation of F-actin nor parasite motility appears to be initiated (Fig.4a,b; Movie_S3).

In summary, this analysis highlights that SLF and CGP act at two temporally different steps during host cell egress. While SLF acts at the initiation step, CGP acts downstream, after the intravacuolar F-actin network has been disassembled.

Finally, we were interested to know if the PV-membrane (PVM) is dissolved. Therefore, we expressed sag1 $1 \Delta \mathrm{GPI}$-dsRed, which is secreted into the PV. Upon lysis of the PV this protein diffuses into the cytosol of the host cell, as seen in case of control parasites (Fig.4c, top row). Using this assay, deletion of CGP had no influence on lysis of the PV, since dsRed signal diffused at a similar time as seen in case of controls. In contrast, upon deletion of SLF, the PVM remained intact and dsRed signal is trapped within the PV (Fig.4c; Movie_S5).

\section{$S L F$ is required for integrity of the $P A$ signalling complex}

Bioinformatic predictions of SLF place this protein into the family of sodium neurotransmitter symporters and demonstrates that it is highly conserved in all apicomplexan parasites.

This protein was previously identified as a putative and dispensable interaction partner of the signalling platform, since knockdown using the auxin-inducible degron (AID) system had no effect on the parasite lytic cycle ${ }^{20}$. However, disruption and excision of slf demonstrated one of the strongest phenotypes obtained in this screen, suggesting that the AID system is insufficient to knockdown protein levels of SLF. Indeed, SLF colocalised with other members 
1 of the PA signalling pathway such as GC, CDC50.1 and UGO (Fig.5a) at the apical tip of the

2 parasite and the intravacuolar network. Importantly, deletion of SLF results in mislocalisation

3 of other components of this signalling complex in the ER and vice versa (Fig.5b,c), indicating

4 that this complex is assembled early in the secretory pathway, probably the ER, and only reaches its final destination if all partners are present. This was seen in $100 \%$ of vacuoles, where one component is missing. While this confirms an important structural role of SLF for functional assembly of the signalling complex, future studies are required to determine if this protein also acts as a sodium neurotransmitter as predicted in ToxoDB. In a first attempt, we focused on GABA ( $\gamma$-aminobutyric acid) as a potential substrate for this putative symporter, since it was demonstrated that $T$. gondii synthesises high levels of GABA $^{21}$ and modulates host cell migration using GABA as messenger ${ }^{22}$. However, we were unable to either complement the phenotype by adding increasing concentrations of GABA or to mimic the phenotype by addition of GABA analogues (Supplementary Fig.10). In conclusion, SLF is a critical component for the integrity of the PA signalling platform required for egress. Future analysis will reveal if this protein is directly involved in the signalling cascade by acting as a symporter.

\section{CGP is a novel component of the conoid}

STED imaging showed a localisation of CGP anterior to the conoid markers RNG2, which localises to the second apical polar ring, and SAS6-like (SAS6L), a marker of the conoid body ${ }^{23}$. This apical localisation was detected in retracted and protruded conoids (Fig.6a). Importantly, conoid structure appeared unaffected upon deletion of $\operatorname{cgp}$ (Fig.6b) indicating that this protein does not play a key role for the integrity of the conoid itself. Similarly, the secretory organelles (micronemes and rhoptries) were not affected by deletion of cgp and secretion of micronemes occurred normally (Fig.3g; Supplementary Fig.8e,f).

While bioinformatic analysis of this protein suggests that it is highly conserved within apicomplexan parasites (not shown), no clear orthologue could be identified in other 
1 eukaryotes. Therefore, future studies are required to identify potential interaction partners and

2 the mechanistic action during host cell egress.

3

4

\section{Discussion}

In this study, we adapted an efficient and tightly regulated conditional Cas9-system based on $\mathrm{sCas} 9^{4}$. Our detailed analysis of this system demonstrated that repair of DSB introduced by Cas9 is occasionally inefficient, leading to aberrant, non-specific phenotypes in a subpopulation of parasites. While these effects need to be taken into account when conducting a phenotypic screen, they can easily be deducted from the readout of the screen, especially when modern, automated image analysis methods are used that are now also available to determine the phenotype of Toxoplasma-Host interactions ${ }^{24}$. Screening of an indicator strain that co-expresses fluorescent actin binding chromobodies $(\mathrm{CbEm})^{5}$ and an apicoplast marker ${ }^{12}$ allowed the identification of mutants with defects in apicoplast maintenance and F-actin dynamics in addition to mutants with inhibited host cell egress. Therefore, we curated a sgRNA library targeting 320 candidate genes that are conserved among apicomplexans. Based on the phenotypic characterisation of identified mutants, we did not identify a novel factor that is directly involved in regulation and organisation of F-actin dynamics during intracellular replication. Instead, several factors were identified, where F-actin dynamics was only slightly altered, when compared to positive controls (Profilin, ADF, Act1). In contrast, two novel genes identified here, slf and cgp, are crucially involved in the regulation actin dynamics during host cell egress where they play distinct roles.

Time-lapse microscopy analysis suggests that disassembly of the intravacuolar network precedes the initiation of motility and egress of parasites from the PV 5 (Movie_S3, S4). Indeed, stabilisation of F-actin by depletion of ADF results in an egress phenotype, where parasites were able to initiate motility, but remain connected by the intravacuolar network (Fig.6c, Supplementary Fig.3).

When we applied this analysis to conditional mutants for SLF and CGP, we observed important differences in the behaviour during host cell egress. While deletion of SLF caused an early 
1 block in host cell egress, where neither the intravacuolar network nor the PVM is disassembled,

2 deletion of CGP caused a late block in egress, where parasites disassembled both the

3 intracellular F-actin network and PVM, but are incapable of initiating motility and leaving the 6 27 host cell. While CGP is not involved in microneme secretion, it is likely that it directly or indirectly regulates gliding motility by regulating F-actin dynamics, since deletion of CGP resulted in clear differences in F-actin dynamics, as evidenced by missing posterior accumulation of F-actin.

In the case of SLF, we found that this protein is an important component of a previously described PA signalling platform required for host cell egress ${ }^{20}$, since deletion of SLF resulted in mislocalisation of the remaining components, CDC50.1, GC or UGO within the ER of the parasite. Together this indicates that the signalling complex needs to be assembled within the ER before being transported to the apical tip and intravacuolar network, where it fulfils its critical function.

These findings highlight the robustness of this approach for finding essential genes based on their function. In future screens, establishment of different indicator lines expressing markers for the secretory organelles, IMC or mitochondria could be used to specifically identify genes involved in organellar biogenesis, parasite replication or maintenance of the endosymbiotic organelles, to name a few examples for future applications of this technology.

Concurrently with our work, Smith and colleagues developed an alternative strategy, based on high-throughput CRISPR-mediated tagging of candidate genes with the AID-system and demonstrate the efficiency of their strategy by labelling and downregulation of the T. gondii kinome, resulting in the characterisation of kinases, involved in diverse functions. Both strategies are highly complementary and have a huge potential to screen the $T$. gondii genome in search for key candidates based on their function. 


\section{Materials and Methods}

\section{Cloning DNA constructs}

The $\mathrm{N}$ - and C-termini of the Cas9 enzyme (split4 variant) were amplified from the original plasmids provided by Zetsche and colleagues ${ }^{4}$ via PCR. The PCR amplicons were ligated into the pGEM®-T Easy vector and sequenced. Subsequently, they were cloned into the Toxoplasma expression vector $\mathrm{p} 5 \mathrm{RT} 70-\mathrm{HX}^{26}$ via the restriction enzymes EcoRI and Pacl. For the C-term-Cas9 vector, the $h x$ selection marker was removed by restriction with Sacll. The resulting plasmids were confirmed by sequencing.

pU6-sag1 gRNA-scaffold and pU6-gap40 gRNA-scaffold sequence was synthesised and cloned into a backbone vector containing the DHFR resistance cassette by GeneScript. The Q5® Site-Directed Mutagenesis Kit (New England Biolabs) was used to insert sgRNAs targeting control genes of interest (act1, adf, frm2 and $d r p A)$ into the synthesised sgRNA plasmid according to the manufacturer's instructions. Importantly, a universal reverse primer was used together with a forward primer to which the whole sgRNA sequenced was attached, see also ${ }^{27}$. Further sgRNAs used in this study were cloned into the universal pU6 vector via Bsal digestion, primers annealing and standard ligation using T4 Ligase (see oligo sequences Supplementary Table 5) as previously described ${ }^{27}$. All sgRNA-plasmids were confirmed by sequencing.

To generate mutated sag ${ }^{\star}$, genomic DNA was amplified and inserted via EcoRI and Pacl into $\mathrm{p} 5 \mathrm{RT} 70-\mathrm{HX}^{26}$. Mutations at the sgRNA binding sequence were introduced via mutagenesis using Q5 Site Mutagenesis following manufacturer's protocol (NEB) using primers described in Supplementary Table 5.

\section{Culturing of T. gondii and host cells}

T. gondii tachyzoites were passaged onto Human foreskin fibroblasts (HFFs; ATCC, SCRC1041) monolayers at $37{ }^{\circ} \mathrm{C}$ and $5 \% \mathrm{CO}_{2}$ in DMEM (Sigma, D6546) supplemented with $10 \%$ FBS (BioSell FBS.US.0500), 4mM L-Glutamate (Sigma, G7513) and $20 \mu \mathrm{g} / \mathrm{ml}$ gentamicin (Sigma G1397). 


\section{Generation of transgenic parasites}

Freshly lysed Toxoplasma tachyzoites were transfected with Amaxa 4D-Nucleofector system (Lonza). $\sim 1 \times 10^{6}$ parasites were centrifuged and resuspended in $50 \mu \mathrm{l} \mathrm{P3}$ buffer. Up to $20 \mu \mathrm{g}$ of DNA for transfection, including vectors, donor DNA and/or single stranded DNA, was ethanol precipitated and resuspended in another $50 \mu \mathrm{l}$ of P3 Buffer. Both resuspensions were mixed in a $100 \mu \mathrm{l}$ cuvette (P3 Primary cells 4D-Nucleofector X kit L, V4XP-3024, Lonza). The programme FI-158 was used for electroporation. Immediately after transfection, parasites were resuspended in fresh complete DMEM and added onto a dish with confluent HFF cells.

For generation of sCas9-parasites, a total of $20 \mu \mathrm{g}$ of the vectors containing the sCas 9 subunits were linearised with Notl and transfected simultaneously in $\mathrm{RH} \Delta h \times g \operatorname{prt}(\mathrm{RH} \Delta \mathrm{HX})$, adding Notl restriction enzyme to the transfection mix. Integrants were selected with $25 \mathrm{mg} / \mathrm{ml}$ mycophenolic acid (MPA) and $40 \mathrm{mg} / \mathrm{ml}$ of xanthine ${ }^{28}$. After isolating a clone containing both functional subunits, sgRNAs against the hxgprt cassette were transfected and the parasites were selected with $80 \mathrm{mg} / \mathrm{ml} 6$-Thioxanthine ${ }^{28}$. The hxgprt cassette was then sequenced to confirm the introduction of indels.

Vectors containing sgRNAs targeting control genes (gap40, sag1, act1, etc) were linearised via Notl and transfected into $\mathrm{RH} \Delta \mathrm{HXsCas9}$ as described above. To select for the $d h f r$ resistance marker, parasites were treated with $1 \mu \mathrm{M}$ pyrimethamine (Sigma 46706; Donald and Roos 1993). Insertion of sgRNAs were confirmed by PCR of the sgRNA cassette (Supplementary Fig.1f).

For the generation of $\mathrm{RHsCas9-CbEm-FNR-RFP,} \mathrm{RHsCas9} \mathrm{parasites} \mathrm{were} \mathrm{firstly} \mathrm{transfected}$ with the vector containing the CbEm cassette ${ }^{5}$. Transfectants were enriched via Fluorescence Assisted Cell Sorting (FACS; S3 BioRad), and a clone was isolated via limiting dilution. Secondly, the vector containing FNR-RFP was linearised via Notl and transfected into RHsCas9-CbEm strain and selected with $20 \mu \mathrm{g} / \mathrm{ml}$ chloramphenicol ${ }^{6}$. After selection, clones were isolated by limiting dilution. 
1 Guide RNAs were synthesised (CustomArray, Inc. USA), amplified by PCR, and cloned as a

2 pool into a plasmid using Gibson Assembly (NEB E26115) as performed by Sidik et al. $(2018)^{15}$. The vector plasmid carries a pU6 promoter, and a DHFR cassette. Assembled plasmids were transformed in two batches, and bacteria allowed to grow to log phase. The plasmids were extracted, purified, pooled, and $200 \mathrm{ng}$ retransformed into the bacteria for further library amplification. Library complexity was estimated based on 1) the number of independent colonies obtained after transforming bacteria with $200 \mathrm{ng}$ of library DNA (as described in Sidik et al. $2018^{15}$ ) and 2) random isolation of individual colonies and sequencing

of the respective sgRNA. From the colony picking, $10 \mu \mathrm{g}$ of different isolated gRNAs were transfected individually into RHsCas9-CbEm-FNR-RFP and analysed in parallel to the screen described below.

Following the generation of the vector library, $60 \mu \mathrm{g}$ of pooled plasmid library was transfected into RHsCas9-CbEm-FNR-RFP line specifically created to be used as an indicator strain for this project. This transfection gave rise to a pool of parasites which were collectively carrying the guide RNA library. A minimum of $1 \times 10^{7}$ parasites were passaged each time. To obtain clonal populations, transfectants were subjected to 3 weeks of pyrimethamine selection followed by FACS (BD FACSAriallI) into ten 96-well plates, one event per well.

\section{Phenotypic screening of sCas9 mutants}

Conditional mutagenesis via sCas9, was induced using $50 \mathrm{nM}$ rapamycin for 48 and $72 \mathrm{~h}$, and the plates were imaged using the LasX navigator on a Leica DMi8 widefield microscope attached to a Leica DFC9000 GTC camera, using a 20x objective. After choosing the correct carrier on the navigator, the plates were aligned and three random images were taken from each well, using the 'On demand' adaptive autofocus setting. The images obtained were independently screened by eye by two investigators before subsequent selection of the candidate clones. Clones which were seen to exhibit aberrant organellar morphologies or altered F-actin dynamics, and egress defects were then selected and the guide RNAs present in the clonal populations, which exhibited phenotypes deemed relevant to the project, were 
1 then sequenced to identify the gene disrupted (Supplementary Fig.4d). To prioritise the

2 candidates for further characterisation, the phenotypes observed were graded from 1 (least

3 severe) to 4 (most severe) (Supplementary table 3).

4

\section{Generation of tagged and floxed strains}

Guide RNAs for cleavage upstream and downstream of the genes of interest were designed using EuPaGDT ${ }^{29}$. Sequences of all sgRNAs employed in this study are detailed in Supplementary Table 1. The sgRNAs were ligated into a vector coding for Cas9-YFP expression, as has been previously described ${ }^{30}$.

Repair templates for integration of a loxP sequence and a tag were generated as in Stortz et al. $(2019)^{11}$. Briefly, repair templates carrying the upstream loxP sequence were ordered as ssDNA oligos (ThermoFischer Scientific), the loxP sequences being flanked by 33 bp of homology.

The repair templates carrying tags, such as 3xHA, SYFP2, Halo and SNAP, were generated by PCR where the 50 bp of homology flanking the tags were introduced via the primer. The repair templates were pooled according to the gene and purified using a PCR purification kit (Blirt) (Supplementary Fig.7a,b).

Parasite transfection, sorting and screening for positive mutants was done according to Stortz et al. (2019) ${ }^{11}$. Briefly, newly released RHDiCre $\Delta k u 80$ tachyzoites ${ }^{31}$ were transfected with the repair templates and 10-12 $\mu \mathrm{g}$ of vectors (encoding Cas-YFP and the respective sgRNAs) as described above. The parasites were mechanically egressed 24 to $48 \mathrm{~h}$ after transfection, passed through a $3 \mu \mathrm{m}$ filter, and those transiently expressing Cas9-YFP enriched via FACS (FACSARIA III, BD Biosciences) into 96-well plates (a minimum of 3 events per well). Resultant clonal lines were screened by PCR and repair template integration confirmed by sequencing (Eurofins Genomics).

For the insertion of CbEm into tagged lines, a specific sgRNA targeting the uracil phosphoribosyltransferase (UPRT) locus was designed and cloned into a Cas9- 
1 YFP-expressing vector. CbEm cassette from the original plasmid used in Periz et al. ${ }^{5}$, was

2 PCR amplified and integrated into the UPRT locus (Supplementary Fig.7k).

3

4

Immunofluorescence assays

Immunofluorescence analysis was carried out as previously described ${ }^{32}$. Briefly, parasites were fixed in $4 \%$ paraformaldehyde for $15-20$ minutes at room temperature. Samples were blocked and permeabilised in phosphate-buffered saline (PBS) with $2 \%$ BSA and $0.2 \%$ Triton $\mathrm{X}-100$ for at least 20 minutes. Antibody labelling was performed using the indicated combinations of primary antibodies for $1 \mathrm{~h}$, followed by the incubation with secondary antibodies for another $45 \mathrm{~min}$. All antibodies are listed in Supplementary Table 6. a-GFPATTO 488 (1:500, Nano Tag Biotechnologies, N0304-At488-L) was directly used for $1 \mathrm{~h}$ after permeablisation. Parasites containing Halo or SNAP tags were incubated with specific dyes for $1 \mathrm{~h}$ and washed away, followed by incubation with media for $1 \mathrm{~h}$ before fixation unless specifically indicated elsewhere (see Supplementary Table 6). Images were taken using Leica DMi8 Widefield microscope or an Abberior 3D STED microscope. The library parasite pictures obtained using the Abberior 3D STED microscope were taken using the confocal setting for FNR-RFP and Hoechst, and STED for the CbEm imaging.

\section{Invasion/replication assays}

$24 \mathrm{~h}$ invasion/replication assays were performed using sCas9 parasite clones isolated as previously described, with some changes ${ }^{32} .5 \times 10^{6}$ parasites were used to infect covers and were left to settle down on ice before allowed invasion for $20 \mathrm{~min}$. Parasites were stained with $\alpha$-SAG1 antibody without permeabilisation and $\alpha$-GAP45 antibody after permeabilisation. For invasion, the number of vacuoles in 10 randomly selected fields of view were counted for each parasite line and condition. For replication, the number of parasites per vacuoles were counted. At least 100 vacuoles were counted.

In the case of RHDiCre $\Delta k u 80$ floxed strains, loxPcgp-Halo parasites were used for invasion/replication assays, loxPslf-Halo were used for invasion assays, and loxPslf-mCherry 
1 were used for replication assays. Parasites were pre-induced for $96 \mathrm{~h} \pm 50 \mathrm{nM}$ rapamycin.

2 Parasites were then mechanically egressed, and $5 \times 10^{6}$ parasites (for invasion assays) or

$34 \times 10^{6}$ (for replication assays) were inoculated in each well and left to invade for $1 \mathrm{~h}$.

4 For invasion assays, parasites were allowed to settle on ice for $10 \mathrm{~min}$, and then allowed to invade for $1 \mathrm{~h}$ at $37^{\circ} \mathrm{C}$ before fixation. Subsequent IFAs were done following the same protocol as the invasion assays done on the sCas9 parasite strains. A minimum of 150 parasites were counted to calculate the percentage of invaded parasites.

For replication assays, samples were washed 3 times with DMEM to remove non-attached parasites and left at $37{ }^{\circ} \mathrm{C}$ for another $24 \mathrm{~h}$. Samples were fixed with $4 \%$ PFA and labelled with a-GAP45 (loxPcgp-Halo) or IMC1 and a-RFP (loxPslf-mCherry).

LoxPcgp-Halo parasites were pre-incubated with HaloTag Oregon Green dye $(0.2 \mu \mathrm{M})$ for $1 \mathrm{~h}$, whereas loxPslf-Halo parasites were incubated with Halo Janelia $646(20 \mathrm{nM})$ for $15 \mathrm{~h}$ prior to the start of the invasion assay.

These experiments were carried out in triplicate and a minimum of 100 parasites/ vacuoles were counted ( $n=>100)$ In case of rapamycin induced floxed parasites (cKO), only parasites/ vacuoles lacking signal for SLF or CGP were included in the counting.

\section{Plaque assay}

A total of 500-1000 parasites per well were inoculated into confluent HFFs in 6 well-plates and incubated for 6 days $\pm 50 \mathrm{nM}$ rapamycin as previously described ${ }^{32}$.

In case of GABA (Tocris, 0344) or Gabapentin (Sigma-Aldrich, G154) plaque assays, media was supplemented with different concentrations of GABA (100 mM stock concentration) or Gabapentin (50 mM stock concentration).

Images were taken using the LAS X Navigator software and a Leica DMi8 Widefield microscope using 10x objective (Microsystems). Starting in the middle of the well, an area of $12 \times 12$ fields was imaged. Focus maps were created and autofocus controls were applied for taking the final images. After acquisition of the images, "mosaic merge" processing tool in LAS $X$ software was used for merging the pictures into one big final image. 
Egress assays

3 For egress assays depicted in Supplementary Fig. 3, induced (50nM Rapamycin) and noninduced $\mathrm{RHsCas9-CbEm-adf/sag1} \mathrm{parasites} \mathrm{were} \mathrm{grown} \mathrm{for} \mathrm{48h.} \mathrm{Egress} \mathrm{was} \mathrm{then} \mathrm{induced} \mathrm{by}$ incubating parasites with $2 \mu \mathrm{M}$ Ci A23187 for 8min under normal culturing conditions. Subsequently, parasites were fixed with 4\%PFA for 20min and $\alpha$-SAG1 or $\alpha$-GAP45 were used for parasite visualisation. $1 \times 10^{5}$ sCas9 background parasites were grown on HFF cells incubated with $\pm 50 \mathrm{nM}$ rapamycin for $4 \mathrm{~h}$. They were then washed with DMEM three times to remove rapamycin and any extracellular parasites. Parasites were maintained for another $44 \mathrm{~h}$ in the incubator at 37 ${ }^{\circ} \mathrm{C}$ before inducing egress. In case of parasites floxed in the DiCre strain, parasites were pre-treated $\pm 50 \mathrm{nM}$ rapamycin for $24 \mathrm{~h}$ and $3 \times 10^{5}$ were inoculated onto confluent HFF cells, incubated for $1 \mathrm{~h}$ at $37^{\circ} \mathrm{C}$, then washed 3 times with PBS and maintained at $37^{\circ} \mathrm{C}$ for $32 \mathrm{~h}$ before inducing egress. Halo tagged parasites were pre-incubated with HaloTag Oregon Green $(0.2 \mu \mathrm{M})$ or Janelia Fluor $646(20$ $\mathrm{nM}$ ) for $1 \mathrm{~h}$ and washed three times with PBS before inducing egress. To induce parasite egress, media was exchanged with pre-warmed DMEM without serum but supplemented with various inducers for different lengths of time ( $2 \mu \mathrm{M}$ Ci A23187 (Sigma-Aldrich, C7522-1mg) for 5 min, $50 \mu \mathrm{M}$ BIPPO (a phosphodiesterase inhibitor that stimulates microneme secretion ${ }^{19}$; Thompson Lab) for $5 \mathrm{~min}$, or $125 \mu \mathrm{M}$ Propranolol hydrochloride (Merck, 40543) for $7 \mathrm{~min}$ ). After induction, sCas9 expressing parasites were fixed with 4\% PFA and the number of egressed and non-egressed vacuoles counted. DiCre-expressing parasites were fixed with either $4 \%$ PFA or $100 \%$ methanol. $\alpha$-SAG1 or $\alpha$-GAP45 antibodies were used for the visualisation of parasites. In case of rapamycin induced floxed parasites, only vacuoles lacking signal of the respective protein was considered in the counting (cKO). At least 100 vacuoles were counted for each condition and replicate and the percentage of the egressed vacuoles was calculated. 
1 For time-lapse images, floxed parasites expressing CbEm (loxPcgp-Halo/CbEm and loxPslf-

$2 \mathrm{Halo} / \mathrm{CbEm}$ ) were treated $\pm 50 \mathrm{nM}$ rapamycin for $24 \mathrm{~h}$ and then mechanically released and

3 inoculated onto glass-bottom live cell dishes, and cultured for a minimum of $32 \mathrm{~h}$ before

$4 \quad$ inducing egress. Halo-tagged parasites were pre-incubated with Janelia Fluor $646(20 \mathrm{nM})$ for

5 around $5 \mathrm{~h}$ followed by washing 3 times with PBS and then incubated with normal media for at least $1 \mathrm{~h}$ before egress induction. Dishes were placed in the pre-warmed chamber of Leica DMi8 microscope and media was exchanged with complete Fluorobrite DMEM (ThermoFischer Scientific, A1896701) containing the respective inducers. Videos were taken with a $63 x$ oil objective at 0.33 frames per second (FPS). Videos were recorded in triplicate per condition as a minimum. In case of conditional knockouts, only vacuoles lacking the signal for SLF or CGP were recorded (cKO).

For calculating the dynamics of $\mathrm{CbEm}$ after stimulating egress chemically, regions of interest (ROI) were drawn around the apicoplast region, the region between basal $\mathrm{CbEm}$ labelling and the apicoplast (termed nuclear region), the T. gondii cell, and a background region outside the vacuole. Relative intensity of the $\mathrm{CbEm}$ in apicoplast region was then determined as:

$$
\text { relative intensity }=\frac{(\text { mean apicoplast }- \text { mean nuclear region }) \times \text { apicoplast area }}{(\text { mean total Toxoplasma }- \text { mean background }) \times \text { total toxoplasma area }}
$$

Relative intensity of the $\mathrm{CbEm}$ in basal pole region was then determined as:

$$
\text { relative intensity }=\frac{(\text { mean basal part }- \text { mean nuclear region }) \times \text { basal part }}{(\text { mean total Toxoplasma }- \text { mean background }) \times \text { total toxoplasma area }}
$$

where mean was defined as:

$$
\text { Mean }=\frac{\text { RawIntDen }}{\text { area }}
$$

To check PVM integrity, loxPcgp-Halo/CbEm and loxPslf-Halo/CbEm were treated with \pm 50 $\mathrm{nM}$ rapamycin for $24 \mathrm{~h}$ and transfected with the vector pTub- sag1 $\Delta \mathrm{GPI}$-dsRed. 48 hours later, 
egress was induced with $50 \mu \mathrm{M}$ BIPPO and recorded as described above. Over 10 egress events were recorded for each condition.

\section{Trail deposition assay and live gliding assay}

For trail deposition assays, induced (50 $\mathrm{nM}$ rapamycin, $72 \mathrm{~h}$ for loxPcgp-Halo and $96 \mathrm{~h}$ for loxPslf-mCherry) and non-induced (72 $\mathrm{h}$ for loxPcgp-Halo and $96 \mathrm{~h}$ for loxPslf-mCherry) parasites were washed, mechanically egressed via 26-gauge needles and filtered through 3 $\mu \mathrm{m}$ filters. Parasites were then centrifuged at $1000 \mathrm{xg}$ for $5 \mathrm{~min}$ at room temperature and the pellet was resuspended in pre-warmed endo buffer (44.7 $\mathrm{mM} \mathrm{K}_{2} \mathrm{SO}_{4}, 10 \mathrm{mM} \mathrm{MgSO}_{4}, 100 \mathrm{mM}$ sucrose, $5 \mathrm{mM}$ glucose, $20 \mathrm{mM}$ Tris, $0.35 \% \mathrm{w} / \mathrm{v}$ BSA, $\mathrm{pH} 8.2$ ) at a concentration of $2 \times 10^{6}$ parasites $/ \mathrm{ml} .1 \mathrm{ml}$ of this mixture was added to a FCS coated glass-bottom live cell dish and incubated for $15 \mathrm{~min}$ at room temperature. Endo buffer was gently replaced with $1 \mathrm{ml}$ of prewarmed sterile gliding buffer (1mM EGTA and 100mM HEPES in HBSS solution). Parasites were incubated for $20 \mathrm{~min}$ at $37{ }^{\circ} \mathrm{C}$ and then fixed with 4\% PFA for $20 \mathrm{~min}$. Parasites were stained with $\alpha$-Toxoplasma gondii antibody (Abcam; see Supplementary table 6 ) without permeabilisation. 15 random fields of view were imaged and the total number of trails parasites left were counted.

For live gliding assays, to measure parasite gliding kinetics, time-lapse videos were taken with a $63 \times$ objective at 2 FPS using a Leica DMi8 microscope using DIC. After 20 minutes of recording per condition, a Z-stack image of the fluorescence channel targeting the protein of interest was taken to distinguish the cKO from the non-induced parasites. Only cKO were considered for the analysis for rapamycin induced parasites. Halo tagged parasites were preincubated with Janelia Fluro 646 dye $(20 \mathrm{nM})$ or HaloTag TMR $(500 \mathrm{nM})$ at least 2 hour as described above before performing live gliding assays. Parasite motility was analysed by manual tracking plugin tool by Icy software. All the assays were done in $\mathrm{Ca}^{2+-}$ free gliding buffer unless otherwise indicated.

For trail deposition and gliding assays with $2 \mu \mathrm{M}$ Ci A23187, compounds were added to the gliding buffer described above. 
Microneme secretion assay

3 Microneme secretion assay protocol was adapted from Bisio et al. (2019) ${ }^{20}$. Parasites treated

4 for $72 \mathrm{~h} \pm 50 \mathrm{nM}$ rapamycin were mechanically released by 26-gauge needles and washed once with cold PBS before being resuspended in pre-warmed intracellular buffer $(5 \mathrm{mM} \mathrm{NaCl}$,

$6142 \mathrm{mM} \mathrm{KCl}, 1 \mathrm{mM} \mathrm{MgCl}$, 2 mM EGTA, $5.6 \mathrm{mM}$ glucose, $25 \mathrm{mM}$ HEPES, pH 7.5). Parasites

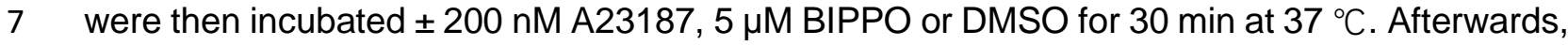

the supernatant was collected and further centrifuged, followed by Western blot analysis (WB).

9 The parasite pellets were washed once with cold PBS followed by centrifugation before performing Western blot analysis. For WB, $6 \%$ stacking and $10 \%$ resolving gel were used. Antibodies used to label the membranes are summarised in Supplementary Table 6. Stained membranes were imaged using Odyssey CLX-1849 (LI-COR).

Imaging processing

All images and movies were processed using Fiji software 2.1.0 and/or Icy Image Processing

Software 1.8.6.0. With the exception of images of parasites expressing $\mathrm{CbEm}$ and time-lapse videos, all widefield images were deconvolved using Huygens essential v18.04.

Data analysis

All data were plotted by Excel or Graphpad Prism 8.2.1.

\section{Data availability}

23 All data and genetic material used for this paper are available from the authors on reasonable request. The source data are provided as a Source Data file.

\section{Author contributions}

W.L. identified egress mutants and performed phenotypic assays for SLF and CGP. J.G. designed and performed the phenotypic screen, identified and tagged candidate genes and 
assisted with phenotyping. J.F.S. established and characterised the sCas9-system in T. gondii, designed the sgRNA library and analysed egress for ADF. M.G. assisted with phenotyping and analysis of parasites. J.P. assisted in experiment design and data analysis. M.M. designed and coordinated the project and experiments, analysed the data, contributed resources and wrote the paper. E.J-R. designed and coordinated the project and experiments, analysed the data, and wrote the paper.

\section{Acknowledgement}

We thank all colleagues, who contributed antibodies and reagents for this study. In particular, we thank the Lourido lab (Whitehead Institute for Biomedical Research) in assisting with the design of the sgRNA library and many useful discussions. W.L. is funded via a CSC fellowship (201806910075). This project is funded within the DFG Priority Programme SPP2225, Project ME 2675/7-1.

\section{Competing interests}

The authors declare no competing interests.

\section{References}

1 Sidik, S. M. et al. A Genome-wide CRISPR Screen in Toxoplasma Identifies Essential Apicomplexan Genes. Cell 166, 1423-1435.e1412, doi:10.1016/j.cell.2016.08.019 (2016).

2 Serpeloni, M. et al. UAP56 is a conserved crucial component of a divergent mRNA export pathway in Toxoplasma gondii. Molecular microbiology 102, 672-689 (2016).

3 Herm-Götz, A. et al. Rapid control of protein level in the apicomplexan Toxoplasma gondii. Nature methods 4, 1003 (2007).

4 Zetsche, B., Volz, S. E. \& Zhang, F. A split-Cas9 architecture for inducible genome editing and transcription modulation. Nature biotechnology 33, 139-142, doi:10.1038/nbt.3149 (2015).

5 Periz, J. et al. Toxoplasma gondii F-actin forms an extensive filamentous network required for material exchange and parasite maturation. elife $\mathbf{6}$, doi:10.7554/eLife.24119 (2017).

6 Kim, K., Soldati, D. \& Boothroyd, J. C. Gene replacement in Toxoplasma gondii with chloramphenicol acetyltransferase as selectable marker. Science 262, 911-914 (1993).

7 Harding, C. R. et al. Gliding Associated Proteins Play Essential Roles during the Formation of the Inner Membrane Complex of Toxoplasma gondii. PLoS pathogens 12, e1005403, doi:10.1371/journal.ppat.1005403 (2016).

8 van Dooren, G. G. et al. A novel dynamin-related protein has been recruited for apicoplast fission in Toxoplasma gondii. Current biology : CB 19, 267-276, doi:10.1016/j.cub.2008.12.048 (2009). 
19 Andenmatten, N. et al. Conditional genome engineering in Toxoplasma gondii uncovers alternative invasion mechanisms. Nature methods 10, 125-127, doi:10.1038/nmeth.2301 (2013).

10 Del Rosario, M. et al. Apicomplexan F-actin is required for efficient nuclear entry during host cell invasion. EMBO Rep, e48896, doi:10.15252/embr.201948896 (2019).

11 Stortz, J. F. et al. Formin-2 drives polymerisation of actin filaments enabling segregation of apicoplasts and cytokinesis in Plasmodium falciparum. eLife 8, doi:10.7554/eLife.49030 (2019).

12 Striepen, B. et al. The plastid of Toxoplasma gondii is divided by association with the centrosomes. The Journal of cell biology 151, 1423-1434, doi:10.1083/jcb.151.7.1423 (2000).

13 Mehta, S. \& Sibley, L. D. Actin depolymerizing factor controls actin turnover and gliding motility in Toxoplasma gondii. Molecular biology of the cell 22, 1290-1299, doi:10.1091/mbc.E10-12-0939 (2011).

14 Plattner, F. et al. Toxoplasma profilin is essential for host cell invasion and TLR11dependent induction of an interleukin-12 response. Cell host \& microbe 3, 77-87, doi:10.1016/j.chom.2008.01.001 (2008).

15 Sidik, S. M., Huet, D. \& Lourido, S. CRISPR-Cas9-based genome-wide screening of Toxoplasma gondii. Nature protocols 13, 307-323, doi:10.1038/nprot.2017.131 (2018).

16 Aquilini, E. et al. An Alveolata secretory machinery adapted to parasite host cell invasion. Nat Microbiol 6, 425-434, doi:10.1038/s41564-020-00854-z (2021).

17 Beck, J. R. et al. A novel family of Toxoplasma IMC proteins displays a hierarchical organization and functions in coordinating parasite division. PLoS pathogens 6, e1001094, doi:10.1371/journal.ppat.1001094 (2010).

18 Andenmatten, $\mathrm{N}$. et al. Conditional genome engineering in Toxoplasma gondii uncovers alternative invasion mechanisms. Nature methods 10, 125-127 (2013).

19 Bullen, H. E., Bisio, H. \& Soldati-Favre, D. The triumvirate of signaling molecules controlling Toxoplasma microneme exocytosis: Cyclic GMP, calcium, and phosphatidic acid. PLoS Pathog 15, e1007670, doi:10.1371/journal.ppat.1007670 (2019).

20 Bisio, H., Lunghi, M., Brochet, M. \& Soldati-Favre, D. Phosphatidic acid governs natural egress in Toxoplasma gondii via a guanylate cyclase receptor platform. Nature microbiology 4, 420-428, doi:10.1038/s41564-018-0339-8 (2019).

21 MacRae, J. I. et al. Mitochondrial metabolism of glucose and glutamine is required for intracellular growth of Toxoplasma gondii. Cell Host Microbe 12, 682-692, doi:10.1016/j.chom.2012.09.013 (2012).

22 Fuks, J. M. et al. GABAergic signaling is linked to a hypermigratory phenotype in dendritic cells infected by Toxoplasma gondii. PLOS Pathog 8, e1003051, doi:10.1371/journal.ppat.1003051 (2012).

23 Koreny, L. et al. Molecular characterization of the conoid complex in Toxoplasma reveals its conservation in all apicomplexans, including Plasmodium species. PLoS biology 19, e3001081, doi:10.1371/journal.pbio.3001081 (2021).

24 Fisch, D. et al. Defining host-pathogen interactions employing an artificial intelligence workflow. eLife 8, doi:10.7554/eLife.40560 (2019).

25 Smith, T. A., Lopez-Perez, G., Shortt, E., Lourido, S. High-throughput functionalization of the Toxoplasma kinome uncovers a novel regulator of invasion and egress. (2021).

26 Meissner, M., Schluter, D. \& Soldati, D. Role of Toxoplasma gondii myosin A in powering parasite gliding and host cell invasion. Science (New York, N.Y.) 298, 837840, doi:10.1126/science.1074553 (2002).

27 Sidik, S. M., Hackett, C. G., Tran, F., Westwood, N. J. \& Lourido, S. Efficient genome engineering of Toxoplasma gondii using CRISPR/Cas9. PloS one 9, e100450, doi:10.1371/journal.pone.0100450 (2014).

28 Donald, R. G., Carter, D., Ullman, B. \& Roos, D. S. Insertional tagging, cloning, and expression of the Toxoplasma gondii hypoxanthine-xanthine-guanine phosphoribosyltransferase gene. Use as a selectable marker for stable transformation. The Journal of biological chemistry 271, 14010-14019, doi:10.1074/jbc.271.24.14010 (1996). 
29 Peng, D. \& Tarleton, R. EuPaGDT: a web tool tailored to design CRISPR guide RNAs for eukaryotic pathogens. Microb Genom 1, e000033, doi:10.1099/mgen.0.000033 (2015).

30 Curt-Varesano, A., Braun, L., Ranquet, C., Hakimi, M. A. \& Bougdour, A. The aspartyl protease TgASP5 mediates the export of the Toxoplasma GRA16 and GRA24 effectors into host cells. Cell Microbiol 18, 151-167, doi:10.1111/cmi.12498 (2016).

31 Hunt, A. et al. Differential requirements for cyclase-associated protein (CAP) in actindependent processes of Toxoplasma gondii. eLife 8, doi:10.7554/eLife.50598 (2019).

32 Egarter, S. et al. The toxoplasma Acto-MyoA motor complex is important but not essential for gliding motility and host cell invasion. PloS one 9, e91819, doi:10.1371/journal.pone.0091819 (2014). 
Figures:

a
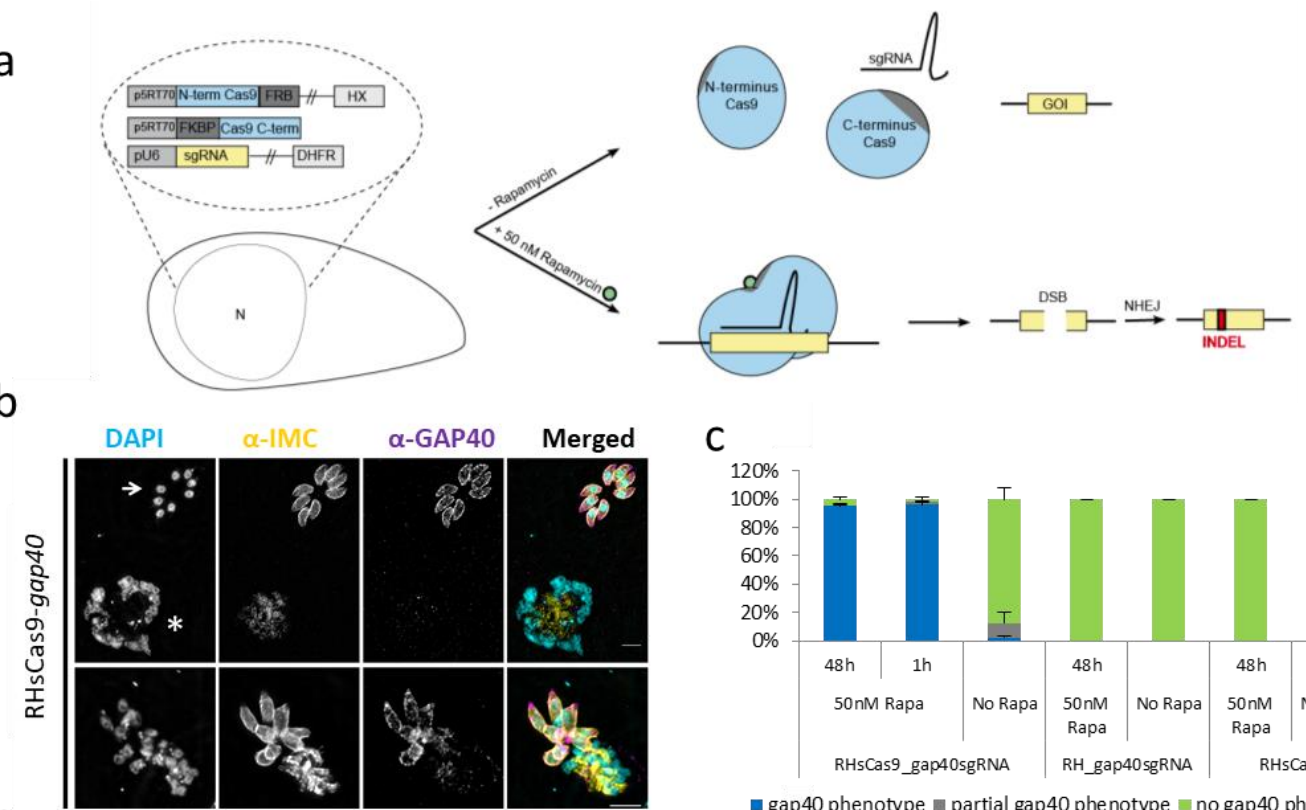

C

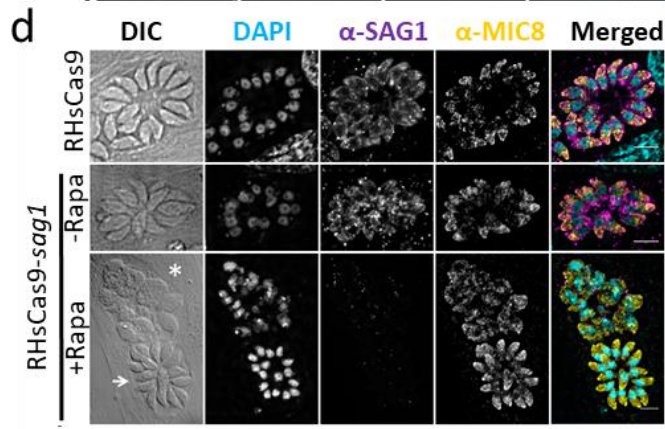

e

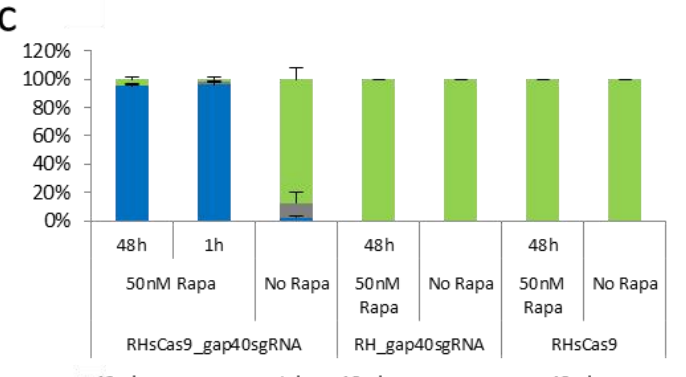

- gap40 phenotype $\|$ partial gap40 phenotype $\square$ no gap40 phenotype
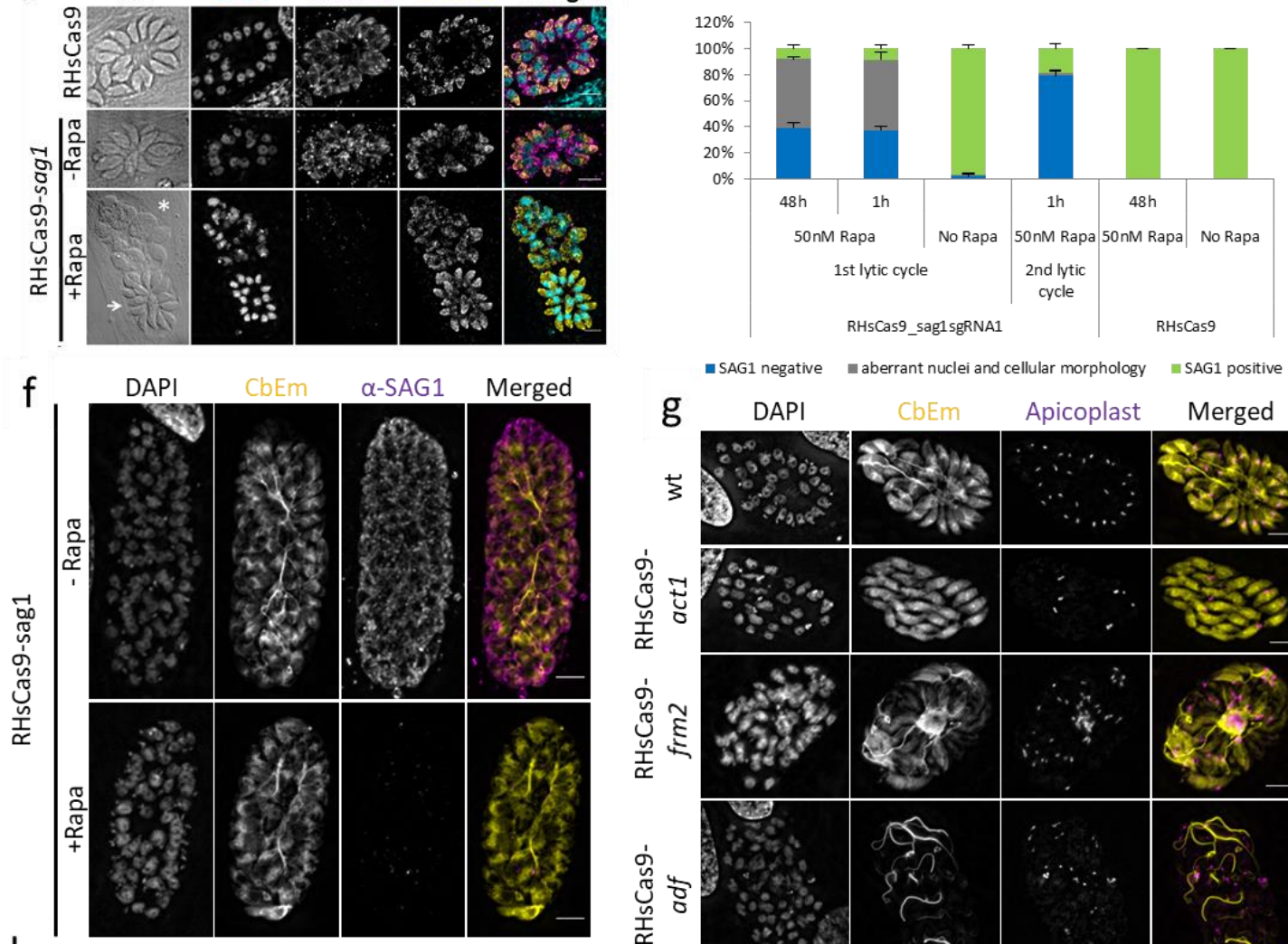

$\mathrm{h}$
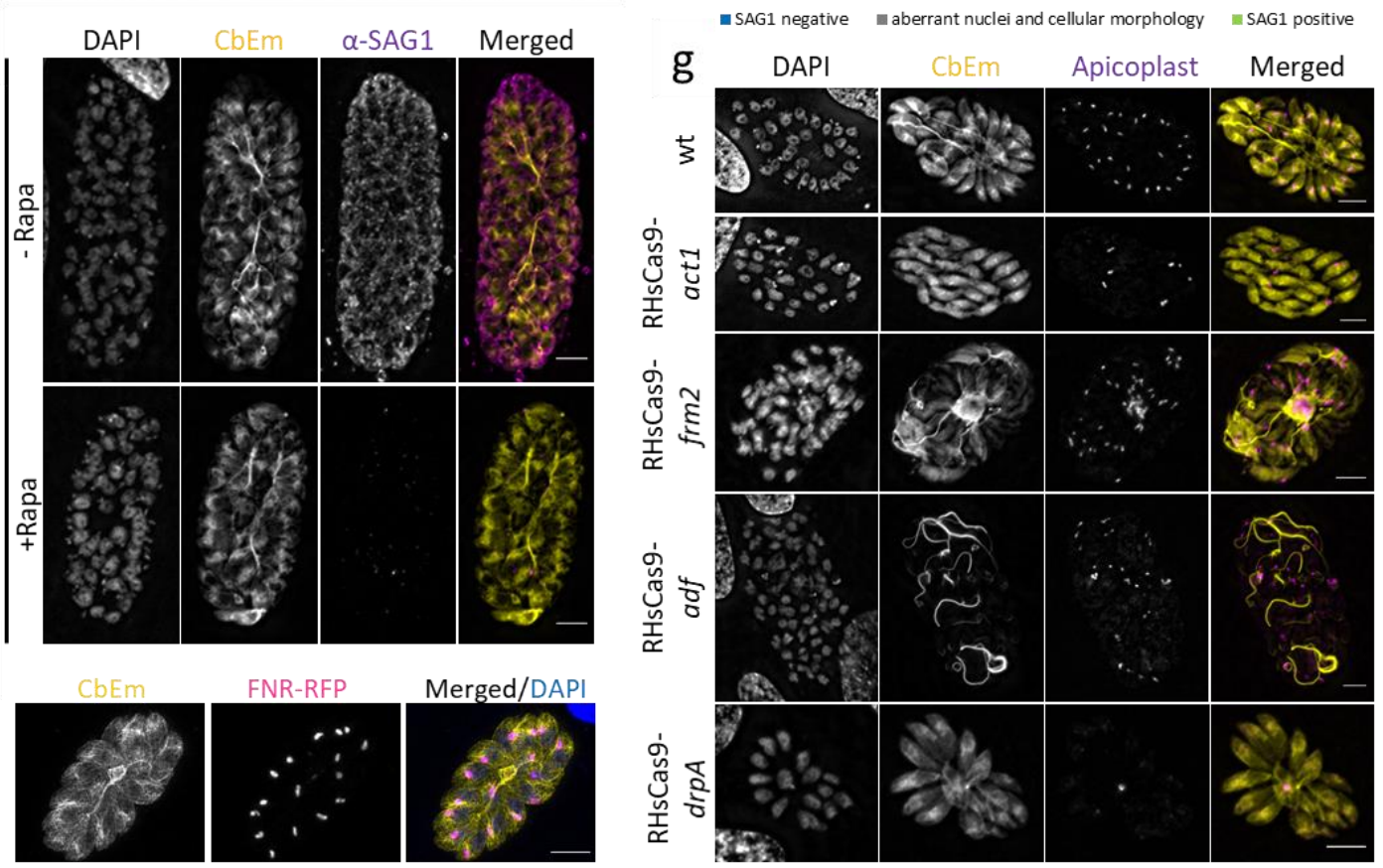

3 Figure 1. Adaptation and characterisation of the splitCas9-system in T. gondii. a, Schematic of the splitCas9-system. Transgenic parasites are generated co-expressing the two splitCas9 subunits together with a single-guide RNA (sgRNA). Upon addition of rapamycin the two subunits dimerise, 6 leading to reconstituted Cas9 activity and therefore to disruption of the gene targeted by the sgRNA. $\mathbf{b}$, 7 Analysis of RHsCas9-gap40 parasites that were treated with $50 \mathrm{nM}$ rapamycin for $48 \mathrm{~h}$ before fixation 
using indicated antibodies. Scale bars are $5 \mu \mathrm{m}$. Three distinct phenotypes can be observed: the gap40 phenotype, as described previously ${ }^{7}$ with collapsed IMC and loss of GAP40 expression (top panel, asterisk); a phenotype where some parasites within the PV are aberrant (bottom panel); and parasites with normal IMC and GAP40 localisation (top panel, arrow). c, Quantification of gap40 phenotypes shown in (a) in indicated parasite strains. Parasites were induced with or without rapamycin for the indicated time and fixed $48 \mathrm{~h}$ post infection. Only parasites expressing both the gap40sgRNA and the sCas9 system presented a gap40 phenotype after induction. Parasites were treated with rapamycin for $1 \mathrm{~h}$ or the whole growth period of $48 \mathrm{~h}$ as indicated. Data represents three independent experiments. For each condition 100 vacuoles were counted (total $n=300$ ). Average and standard deviation (SD) are represented. $\mathbf{d}$, Analysis of $\mathrm{RHsCas9-sag1}$ parasites that were treated with $50 \mathrm{nM}$ rapamycin for $48 \mathrm{~h}$ before fixation using indicated antibodies. Nuclei were stained with DAPI. Scale bars are $5 \mu \mathrm{m}$. Three distinct phenotypes can be observed: healthy vacuoles lacking SAG1 (bottom panel, arrow); and parasites lacking SAG1 while displaying aberrant nuclear and cellular morphology (bottom panel, asterisk). e, Quantification of the phenotypes shown in (d). Aberrant nuclei and cellular morphology were observed only when sag1 was disrupted (KO) by $\mathrm{sCas} 9$ activation ( $1^{\text {st }}$ lytic cycle). Abundance of non-healthy parasites was reduced to

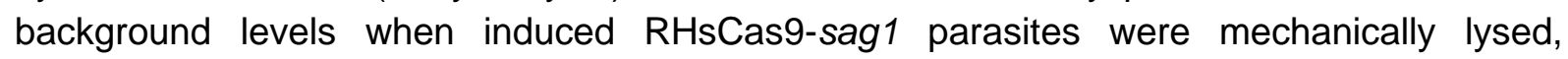
transferred onto fresh host cells and grown again for $48 \mathrm{~h}$ in a second lytic cycle $\left(2^{\text {nd }}\right.$ generation; total incubation of $96 \mathrm{~h}$ ). Parasites were treated with rapamycin for $1 \mathrm{~h}$ or for the whole growth period of $48 \mathrm{~h}$ as indicated. Data represent three independent experiments. For each condition, 100 vacuoles were counted (total $n=300$ ). Average and standard deviation (SD) are represented. $\mathbf{f}$, Analysis of indicator parasites expressing sgRNA targeting sag1. Disruption of sag1 has no effect on the F-actin network. Scale bars are $5 \mu \mathrm{m}$. $\mathbf{g}$, Analysis of indicator parasites expressing indicated sgRNAs. IFA depicting the effect of $\operatorname{drpA}(\mathrm{RHsCas9-} d r p A)$, or adf (RHsCas9-adf), act1 (RHsCas9-act1) and frm2 (RHsCas9-frm2) disruption on actin network and apicoplast segregation. To achieve gene disruption (KO), parasites were incubated with $50 \mathrm{nM}$ rapamycin for $1 \mathrm{~h}$ and fixed after 48h.Nuclei were stained with DAPI. Scale bars are $5 \mu \mathrm{m}$. $\mathbf{h}$, Depiction of indicator parasites co-expressing CbEm, FNR-RFP and the sCas9-subunits. Scale bar is $5 \mu \mathrm{m}$. 

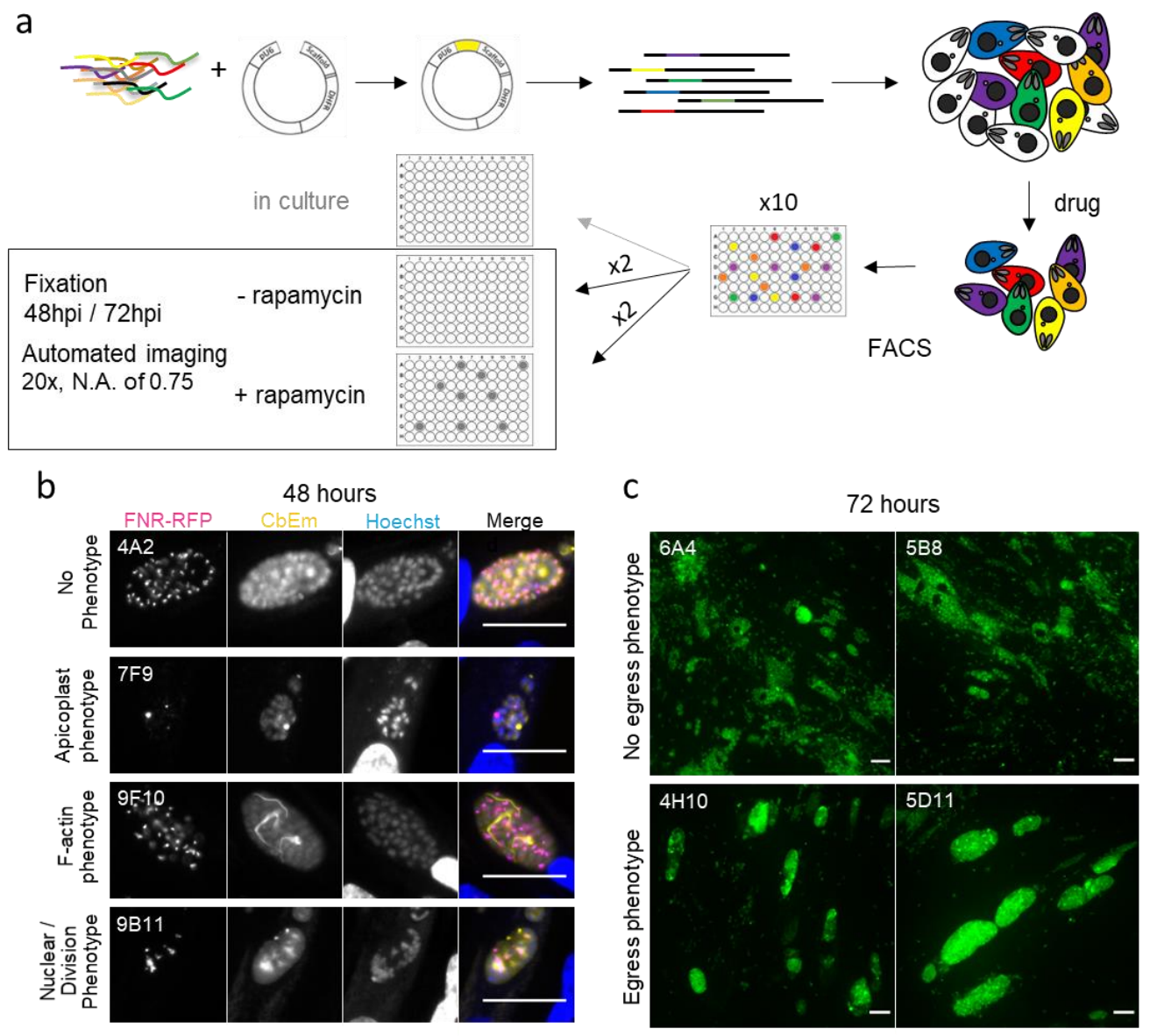

d 608 clones

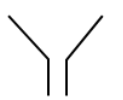

267 clones selected

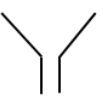

99 unique candidates identified<smiles>CC=CC</smiles>

42 candidates for validation<smiles>CC1CCCCC1</smiles>

2 candidates for characterisation

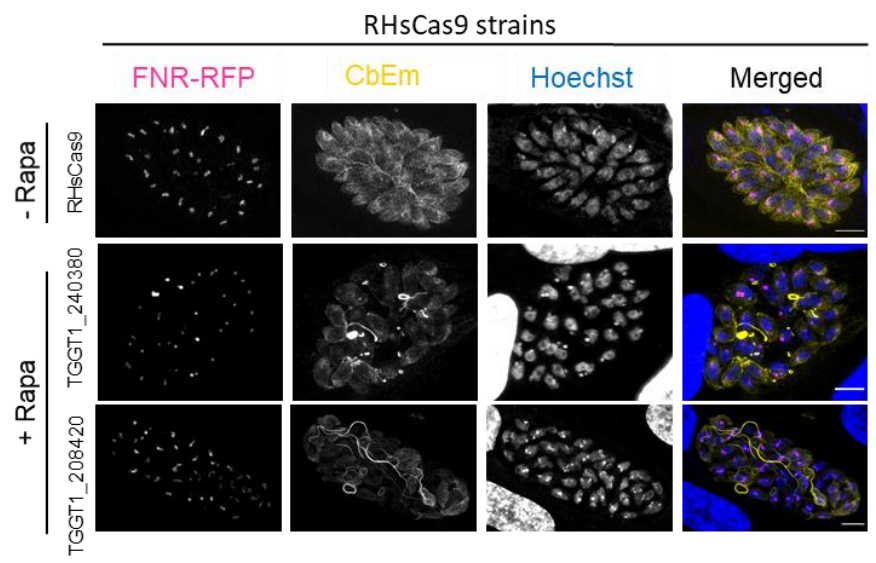

Figure 2. Phenotypic screen for actin dynamics, apicoplast segregation and egress mutants. a, Scheme of the experimental design. Indicator parasites, RHsCas9-CbEm-FNR-RFP, were transfected with the sgRNA library and grown in human foreskin fibroblasts (HFFs). Parasites were selected with pyrimethamine and sorted into 96-well plates. After 7 days, they were split into replica plates. Two replica plates were induced for $48 \mathrm{~h}$ or $72 \mathrm{~h}$ with rapamycin, before automated imaging was performed. Candidate clones were subsequently picked from non-induced replica plates. b, Representative images of observed phenotypes $48 \mathrm{~h}$ post induction. Scale bars are $25 \mu \mathrm{m}$ c, Representative images $72 \mathrm{hpi}$. Top row shows clones without egress phenotype. Bottom row shows two examples of clones with a strong egress phenotype. Scale bars, $30 \mu \mathrm{m} \mathrm{d}$, Schematic representation of the selection of candidate genes (left) and phenotype of the two identified egress mutants $48 \mathrm{hpi}$. Note that the apicoplast appears normal, while slight differences in the F-actin network, which appears more prominent and condensed, 
bioRxiv preprint doi: https://doi.org/10.1101/2021.09.24.461619; this version posted September 24, 2021. The copyright holder for this preprint (which was not certified by peer review) is the author/funder. All rights reserved. No reuse allowed without permission.

1 can be observed. Parasites were induced for $48 \mathrm{~h}$ with rapamycin before fixing and imaging. Scale bars, $25 \mu \mathrm{m}$. 
a

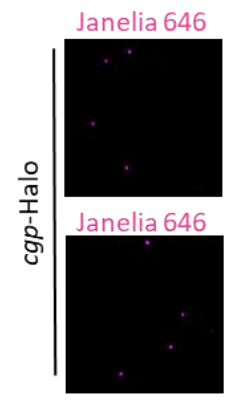

b

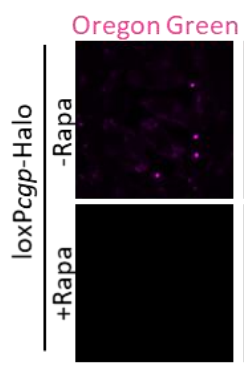

C

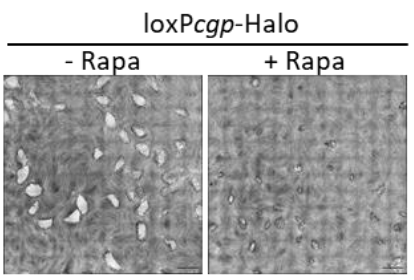

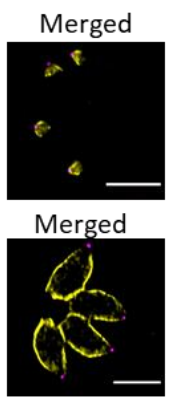
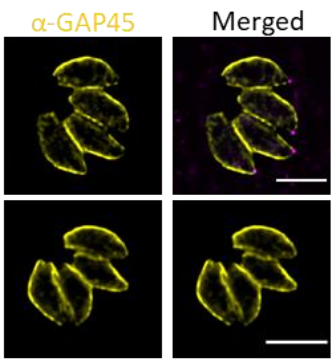
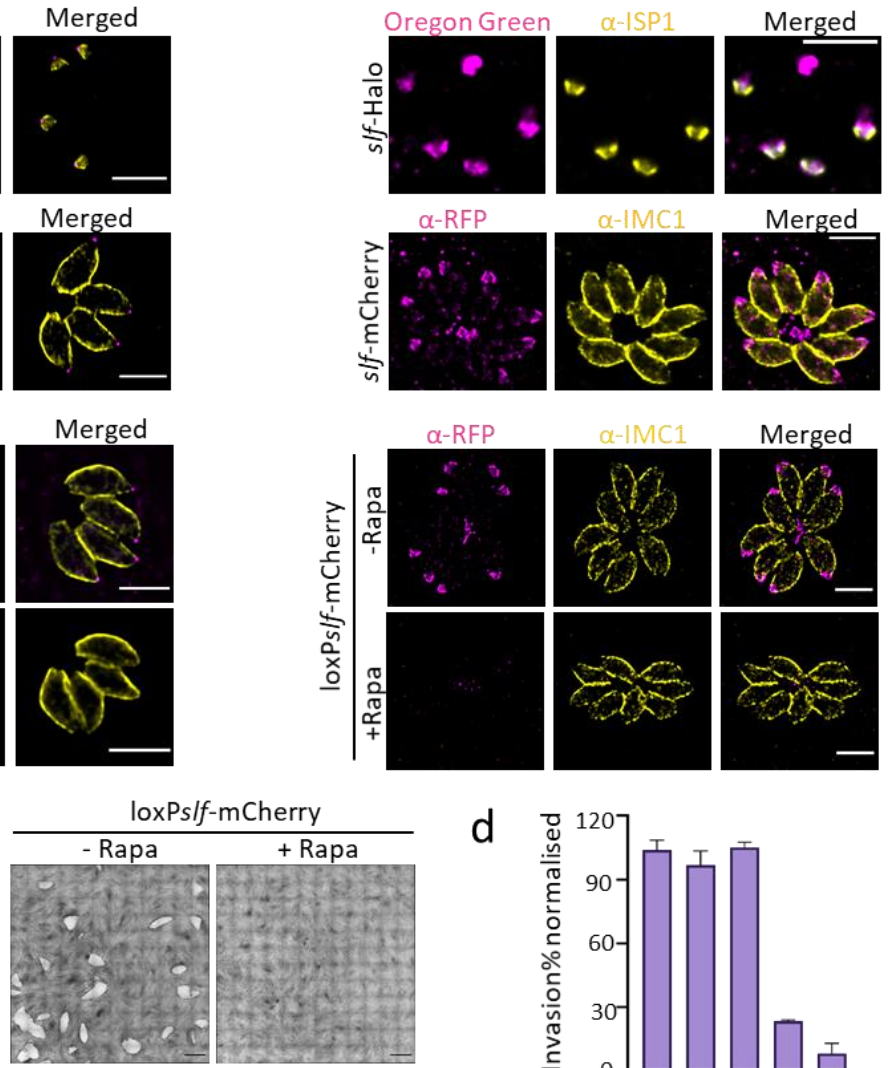

e

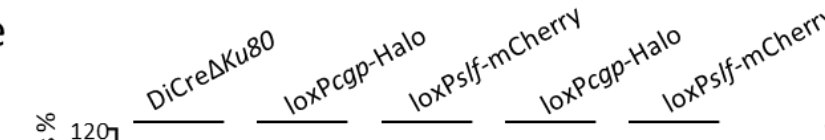

d

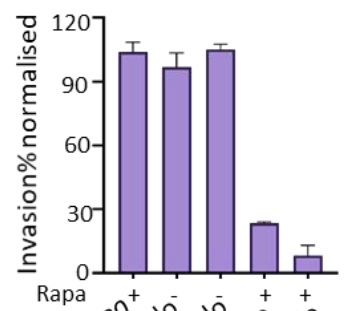

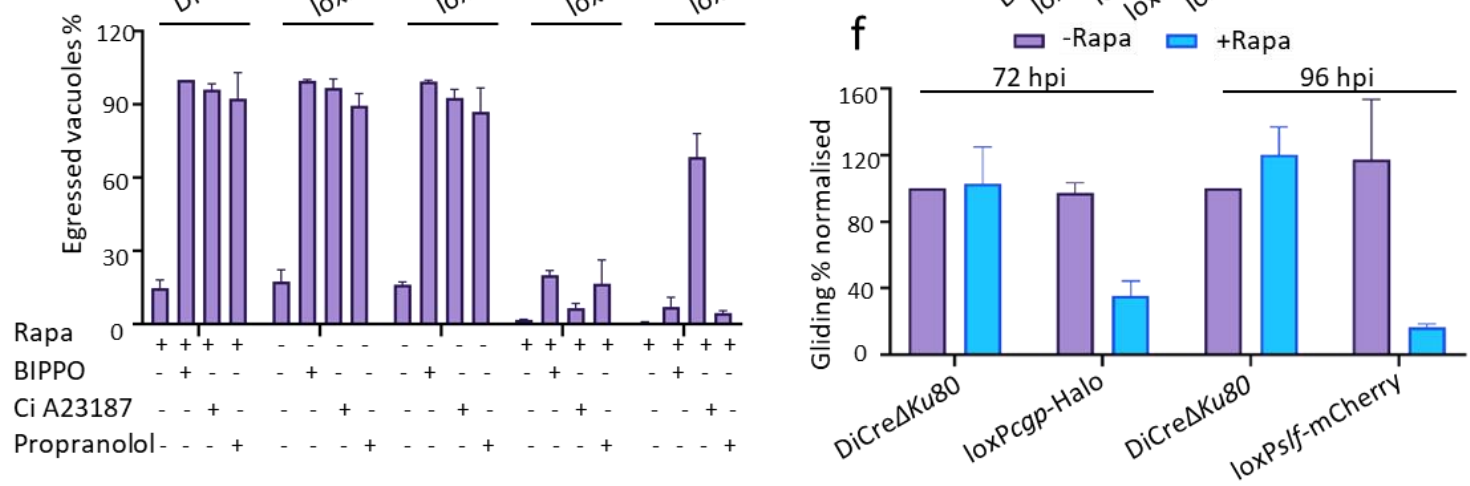
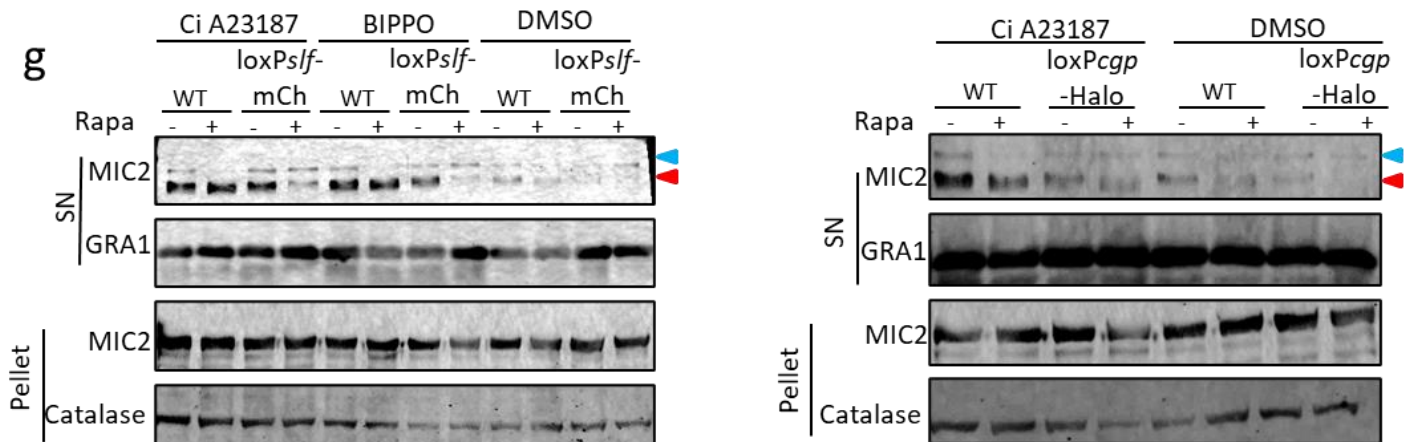

Figure 3. Analysis of cgp and slf. a, Endogenously-tagged TGGT1_240380 (cgp-Halo) localised at the apical tip, while TGGT1_208420 (slf-Halo or slf-mCherry) demonstrated a dual localisation at the apical region and the residual body. Dual labelling was performed to stain the apical cap (ISP1) of the parasite. Scale bar: $5 \mu \mathrm{m}$. b, Analysis of conditional knockouts for cgp and slf using the DiCre-system. IFA depicts floxed cgp-Halo (loxPcgp-Halo) and floxed s/f-mCherry (loxPslf-mCherry) induced or not with $50 \mathrm{nM}$ rapamycin (Rapa). 72 hours post induction, both proteins were not detectable by IFA. Dual 
1 labelling was performed to stain the IMC (GAP45 or IMC1) of the parasite, which shows no differences 2 upon deletion of slf or cgp. Scale bar, $5 \mu \mathrm{m}$. c, Plaque assays of loxPcgp-Halo and loxPslf-mCherry 3 parasites confirm a severe growth defect upon deletion of slf or cgp. Parasites were treated $\pm 50 \mathrm{nM}$ 4 rapamycin for 6 days before fixation. Scale bar, $1.5 \mathrm{~mm}$. $\mathbf{d}$, Invasion-attachment assay for the indicated 5 parasites lines. Results were normalised to DiCre $\Delta k u 80$ strain. This assay was performed in triplicate. 6 Bars represent mean \pm standard deviation (SD). For each condition, at least 150 vacuoles were counted 7 (total $\mathrm{n} \geq 450$ ). e, Induced egress assay in the presence or absence of different inducers: Calcium ionophore (Ci) A23187 $(2 \mu \mathrm{M})$ for $5 \mathrm{~min}$, BIPPO $(50 \mu \mathrm{M})$ for $5 \mathrm{~min}$, and Propranolol $(125 \mu \mathrm{M})$ for $7 \mathrm{~min}$. Experiment was performed in triplicates. Data shown as Mean \pm SD. For each condition 100 vacuoles were counted (total $n=300$ ). $\mathbf{f}$, Quantification of trail deposition. Results were normalised to DiCre $\Delta k u 80$ strain. 3 biological replicates were performed. Data were presented as mean \pm SD. g, Microneme secretion assay was perform on wildtype (WT) parasites, loxPcgp-Halo and loxPslf-mCherry (loxPslf$\mathrm{mCh}$ ). While parasites lacking CGP showed normal microneme secretion comparable to WT, s/f cKO parasites had a decreased secretion. Triangles indicate the unprocessed (blue) and processed (red) form of MIC2. This assay was performed in 3 biological replicates. Representative images are shown. 
a

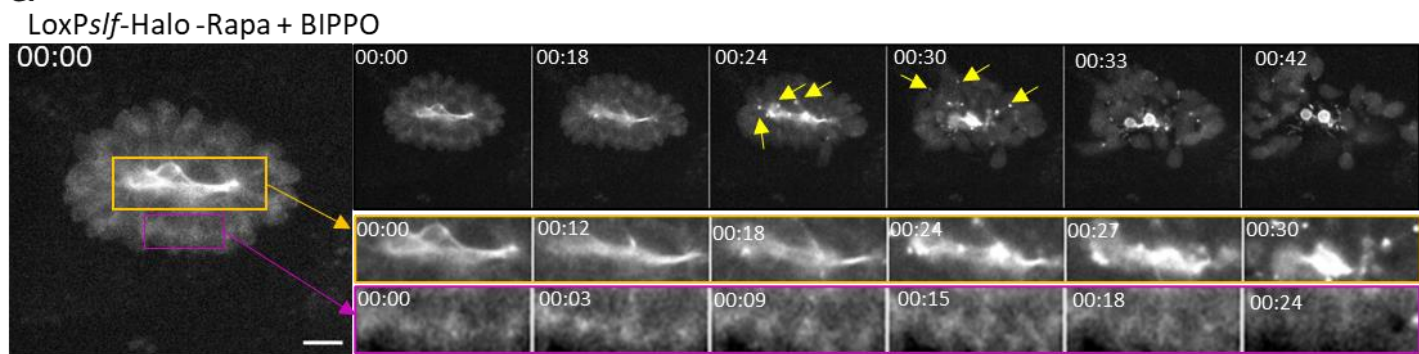

LoxPcgp-Halo (cKO) +Rapa +BIPPO


C LoxPcgp-Halo-Rapa + BIPPO
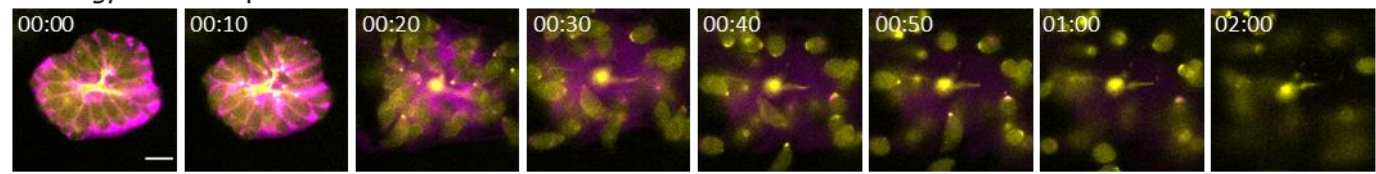

LoxPcgp-Halo (cKO) +Rapa +BIPPO
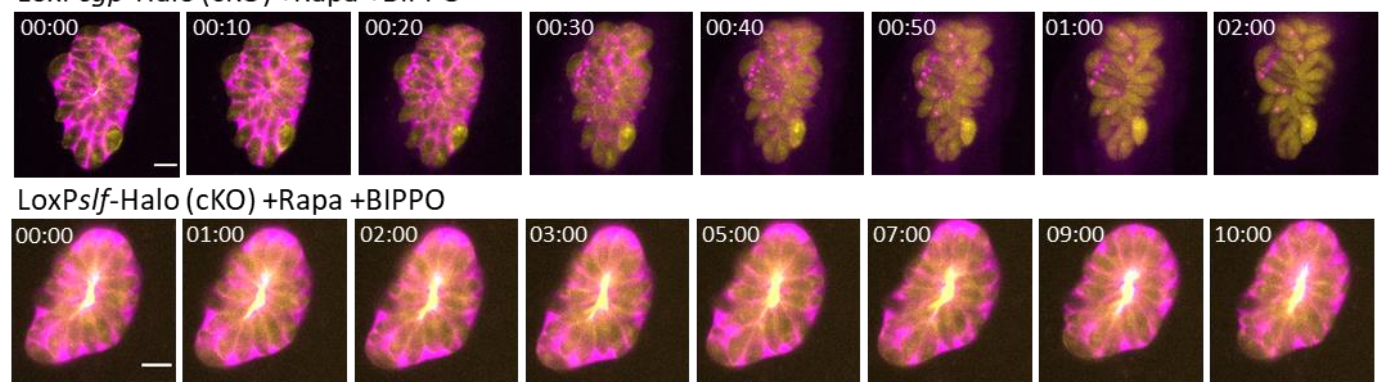

Figure 4. SLF and CGP act at different times during egress. a, Egress of parasites expressing CbEm labelling F-actin was induced with $50 \mu \mathrm{M}$ BIPPO and imaged with an interval of 3 seconds between frames. yellow boxes show F-actin in intravacuolar network. Pink boxes show F-actin polymerisation centre close to the apicoplast/Golgi region (insets have enhanced contrast for better visualisation). Upper panel showing non-induced parasites as control (see movie_S4). F-actin disintegrated rapidly upon addition of BIPPO accompanied with F-actin accumulation at the basal pole (indicated by yellow arrows). The polymerisation centre close to the apicoplast/Golgi region appeared to become inactive 
during egress. Middle panel depicting F-actin in loxPcgp-Halo parasites previously induced with $50 \mathrm{nM}$ rapamycin. Only parasites lacking signal for CGP were recorded (see movie_S3). Network disintegration and inactivation of the F-actin polymerisation centre close to the apicoplast/Golgi appeared normal, while parasites remained immotile and no relocalisation of F-actin to the basal pole is evident (blue arrow). Bottom panel depicting F-actin in loxPslf-Halo parasites previously induced with $50 \mathrm{nM}$ rapamycin. Only parasites lacking signal for SLF were recorded (see movie_S4). The F-actin network remained intact and the F-actin polymerisation centre close to the apicoplast/Golgi remained active. Time is displayed in minutes : seconds. Scale bar, $5 \mu \mathrm{m}$. b, Quantification of the average relative fluorescence intensity of indicated parasites after induction of egress with $50 \mu \mathrm{M}$ BIPPO. The graphs show the average of indicated individual measurements. Red arrows indicate the time where the F-actin network started to depolymerise. In control parasites disassembly of the F-actin network was followed by a reduction of polymerisation at the centre close to the apicoplast/Golgi region and an increase accumulation of F-actin at the basal pole of the parasite. Deletion of cgp resulted in similar behaviour of the F-actin network and comparable reduction at the polymerisation centre close to the apicoplast/Golgi, but no accumulation of $\mathrm{F}$-actin at the basal pole. Parasites lacking slf showed no reorganisation of $\mathrm{F}$ actin upon stimulation of egress with BIPPO. Time interval between each frame is 3 seconds. c, Egress of parasites expressing CbEm (yellow) and SAG1 $\triangle$ GPI-dsRed (pink) was induced with $50 \mu \mathrm{M}$ BIPPO. Upper panel showing non-induced parasites, upon induction, dsRed signal diffused into the host cell, indicating lysis of parasitophorous vacuole membrane (PVM). Middle panel depicting parasites lacking CGP signal after rapamycin induction. Although the PVM lysed, parasites did not move out of the cell. Bottom panel depicting parasites lacking SLF signal after rapamycin induction, where dsRed signal is kept within the PV suggesting intact PVM. Time is displayed in minutes : seconds. Scale bar, $5 \mu \mathrm{m}$. See also movies_S5. 
a

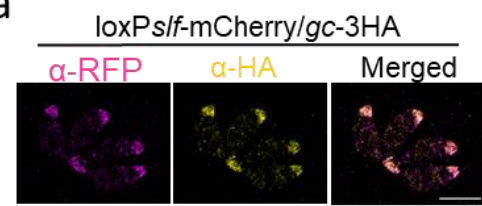

b

b loxPs/f-mCherry/gc-3HA
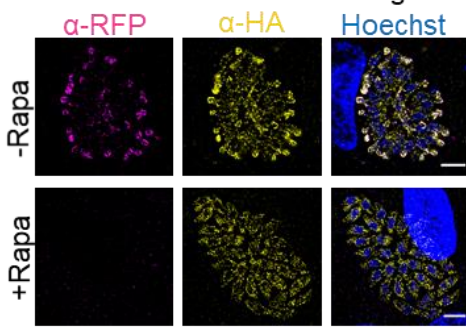

C
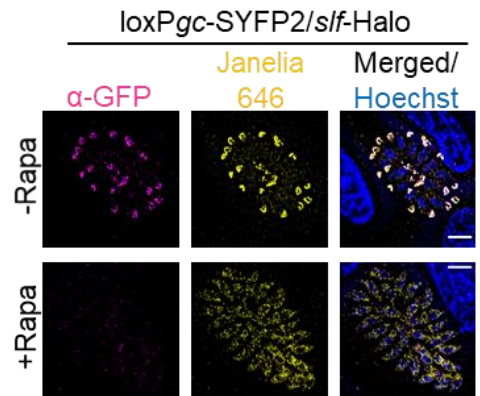

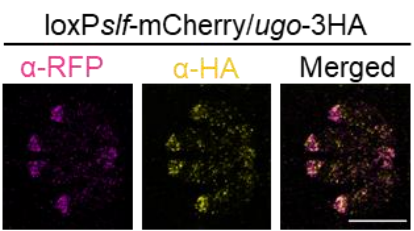

$\frac{\text { loxPslf-mCherry/ugo-SYFP2 }}{\text { Merged/ }}$
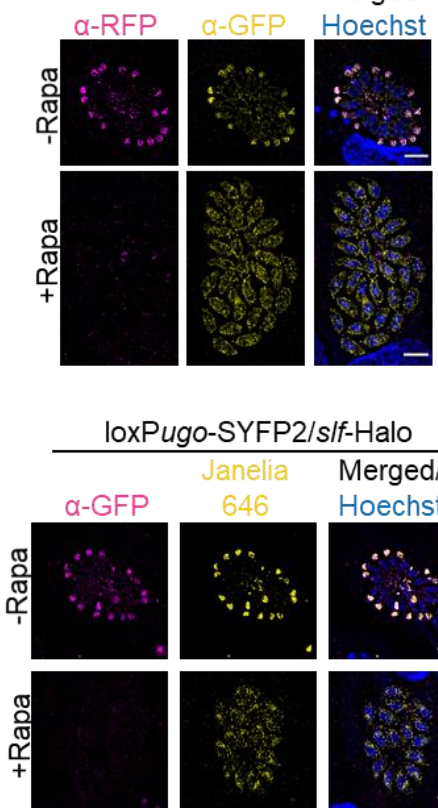

loxPs/f-mCherry/cdc50.1-SYFP2

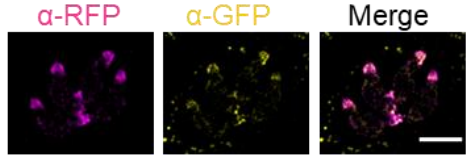

$\frac{\text { loxPslf-mCherry/cdc50.1-SYFP2 }}{\text { Merged/ }}$
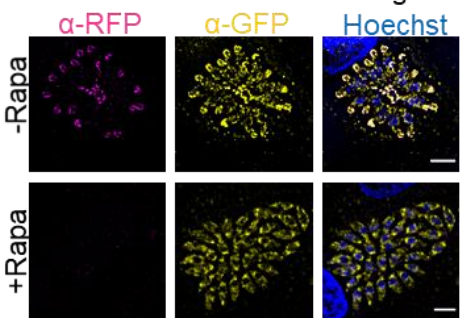

Figure 5. SLF is a crucial part of the signalling platform. a, GC, UGO and CDC50.1 colocalise with SLF. Scale bar: $5 \mu \mathrm{m}$. b, Localisation of indicated components of the signalling platform upon deletion of slf. Deletion of slf-mCherry results in mislocalisation of GC/UGO/CDC50.1. Analysis was performed 96 hours post induction. Scale bar, $5 \mu \mathrm{m}$. c, Deletion of $\mathrm{gc} / \mathrm{ugo} / \mathrm{cdc} 50.1$ (see also Supplementary Fig. 7 for generation of conditional KOs for individual components) results in mislocalisation of SLF at 96 hours post induction. Scale bar: $5 \mu \mathrm{m}$. 
a

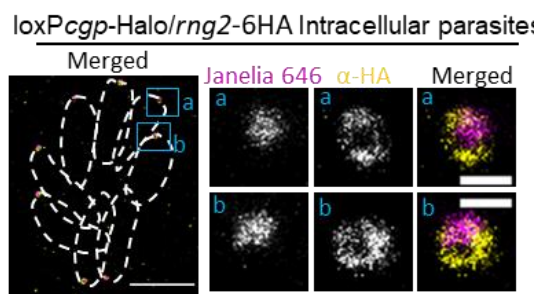

loxPcgp-Halo/sas6l-eGFP Intracellular parasites
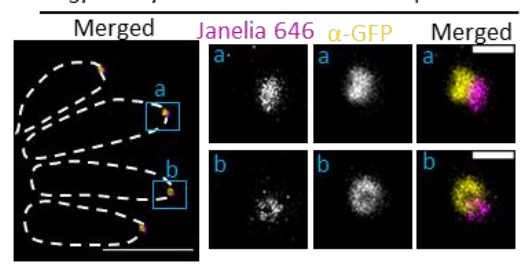

b

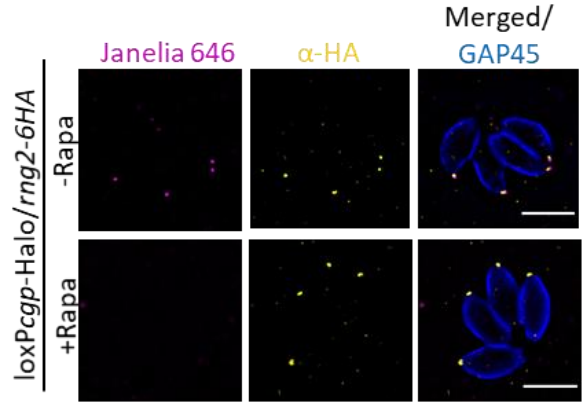

loxPcgp-Halo/rng2-6HAExtracellular parasites

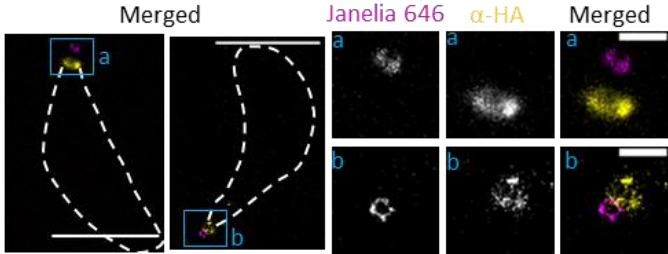

loxPcgp-Halo/sas6/-eGFP Extracellular parasites

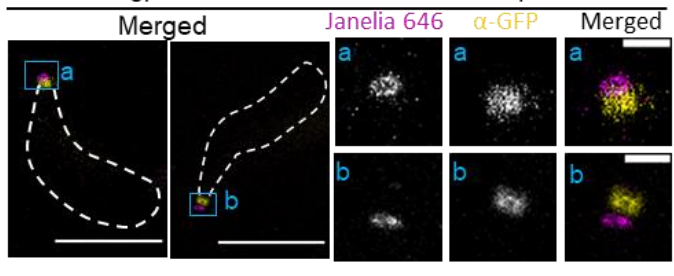

Merged/

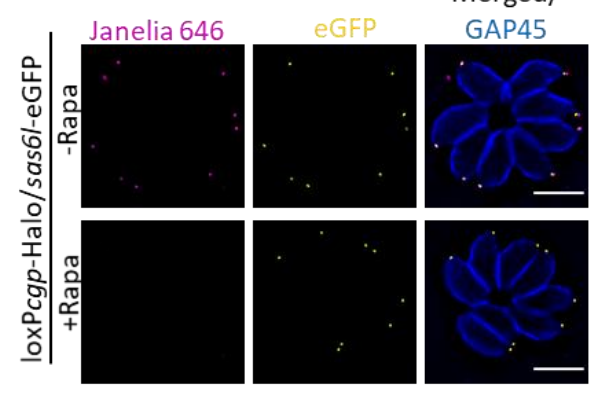

C
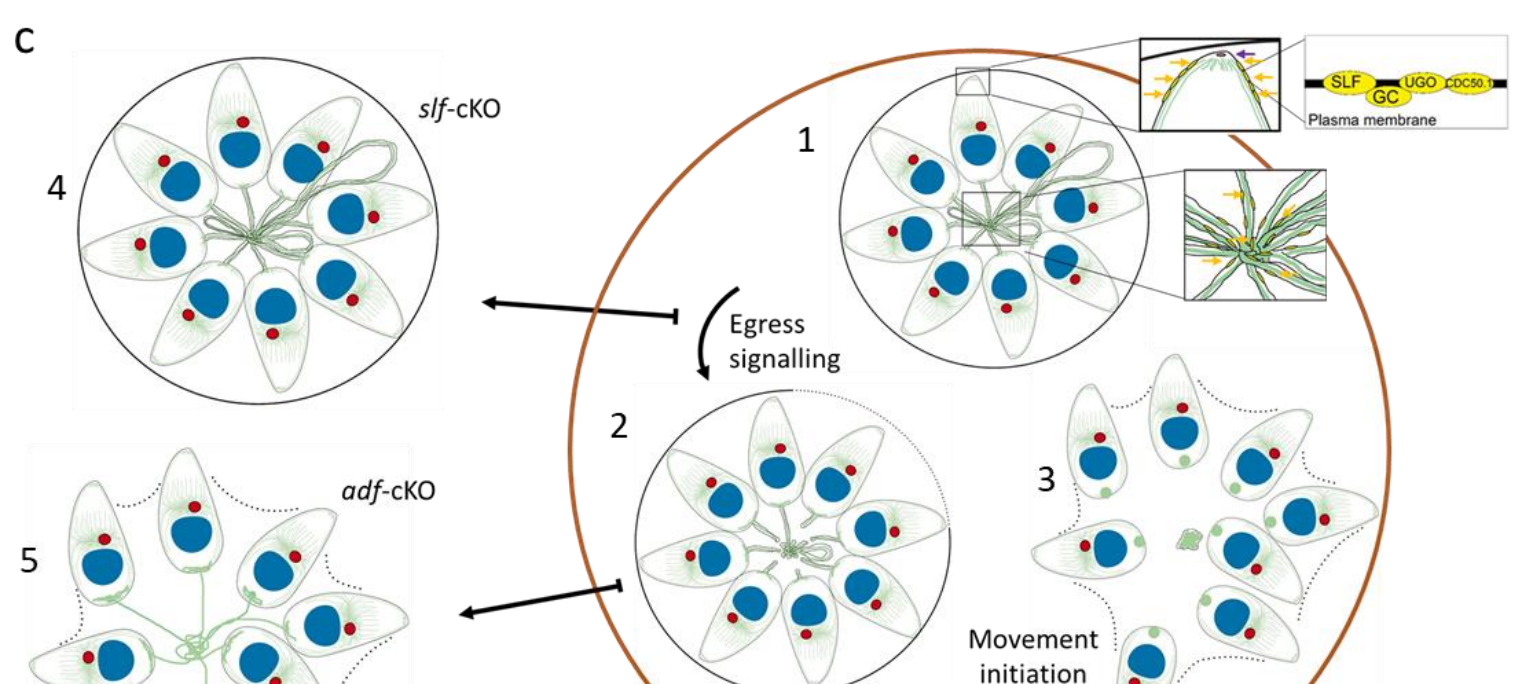

Figure 6. CGP localises to the preconoidal rings and egress models. a, STED microscopy of indicated parasites. Colocalisation of CGP-Halo with RNG2 or SAS6L proteins in intracellular parasites or extracellular parasites following conoid protrusion stimulation with $2 \mu \mathrm{M}$ calcium ionophore A23187 for $10 \mathrm{~min}$ before fixation. White dash lines indicate parasite periphery. Scale bar, $5 \mu \mathrm{m}$ for intracellular parasites and $3 \mu \mathrm{m}$ for extracellular parasites. Scale bar of insets: $0.5 \mu \mathrm{m}$. $\mathbf{b}$, Absence of CGP did not 
affect the localisation of RNG2 and SAS6L. Scale bar, $5 \mu \mathrm{m}$. c, Block of egress caused by deletion of SLF, CGP and ADF (see Supplementary Fig.3) is intrinsically linked to the disassembly of the F-actin network. 1-3) Natural egress process in a wildtype parasite. 1) At the start of the egress the signalling platform consisting of CDC50.1, GC, UGO and SLF, localised at the plasma membrane of the apical tip and the intravacuolar network (IVN), initiates a cascade leading to egress. Zoom in boxes: yellow arrows indicate the position of the signalling platform at the apical tip and residual body. Purple arrow indicates the position of CGP at the conoid of the parasites. 2) As a first step towards egress, the IVN disassembles, which coincides with lysis of the PVM. 3) F-actin accumulates at the basal end of the parasite and motility is initiated. 4) In the absence of SLF, the signalling platform is mislocalised and therefore not functional, resulting in early block of egress. No disassembly of the intravacuolar network or PVM lysis occurs. 5) Depletion of actin regulatory proteins, such as ADF results in stabilisation of the network. Although parasites lyse the PVM and initiate motility, the network keeps connecting individual parasites resulting in delayed or blocked egress (see Figure S3 and ${ }^{11}$ ). 6) Deletion of cgp results in a late block of egress. The signalling cascade appears intact, leading to disassembly of the intravacuolar network and lysis of the PVM. Instead, no relocation of F-actin to the basal pole can occur and motility is not initiated. 